\title{
SOLUTIONS IN TERMS OF INTEGRALS OF MULTIVALUED FUNCTIONS FOR THE CLASSICAL HYPERGEOMETRIC EQUATIONS AND THE HYPERGEOMETRIC SYSTEM ON THE CONFIGURATION SPACE
}

\author{
Katsuhisa MIMACHI and Masatoshi NOUMI
}

(Received 27 October 2015)

\begin{abstract}
The systems of differential equations associated with the classical hypergeometric functions and the hypergeometric functions on the space of point configurations are investigated from the viewpoint of the twisted de Rham theory. In each case, it is proved that the integral of a certain multivalued function over an arbitrary twisted (or loaded) cycle satisfies the system of differential equations in question. The classical hypergeometric functions studied here include Appell's hypergeometric functions $F_{1}, F_{2}, F_{3}, F_{4}$, Lauricella's hypergeometric functions $F_{A}, F_{B}, F_{C}, F_{D}$, and the generalized hypergeometric function ${ }_{n+1} F_{n}$.
\end{abstract}

\section{Introduction}

In this paper, we investigate the systems of differential equations associated with the classical hypergeometric functions, as well as the hypergeometric functions on the space of point configurations, in the framework of the twisted de Rham theory. In each case, we prove that the integral of a certain multivalued function over an arbitrary twisted (or loaded) cycle satisfies the system of differential equations in question. The classical hypergeometric functions studied here include Appell's hypergeometric functions $F_{1}, F_{2}, F_{3}, F_{4}$, Lauricella's hypergeometric functions $F_{A}, F_{B}, F_{C}, F_{D}$, and the generalized hypergeometric function ${ }_{n+1} F_{n}$. The corresponding differential equations will be denoted by $E_{1}, E_{2}, E_{3}, E_{4}, E_{A}, E_{B}, E_{C}, E_{D}$, and ${ }_{n+1} E_{n}$.

We briefly explain the point of our investigation, taking the example of integrals associated with $_{n+1} F_{n}$ :

$$
\int_{D} u(x ; t) d t_{1} \cdots d t_{n}, \quad u(x ; t)=\prod_{i=1}^{n} t_{i}^{\alpha_{i+1}-\beta_{i}} \prod_{i=1}^{n+1}\left(t_{i-1}-t_{i}\right)^{\beta_{i}-\alpha_{i}-1}
$$

2010 Mathematics Subject Classification: Primary 33C60, 33C65, 33C20.

Keywords: hypergeometric integrals; classical hypergeometric equations; classical hypergeometric functions; Appell's hypergeometric functions $F_{1}, F_{2}, F_{3}, F_{4}$; Lauricella's hypergeometric functions $F_{A}, F_{B}, F_{C}, F_{D}$; generalized hypergeometric function ${ }_{n+1} F_{n}$; hypergeometric function on the configuration space.

(c) 2016 Faculty of Mathematics, Kyushu University 
where $t_{0}=1, t_{n+1}=x, \beta_{n+1}=1$. It is shown in [M1, Proposition 2.1] that, when the variable $x$ is real and $-\infty<x<0$, the integrals (0.1) over the real domains

$$
D_{i}^{(0)}=\left\{x<t_{n}<\cdots<t_{i}<0,1<t_{1}<\cdots<t_{i-1}<+\infty\right\}, \quad i=1, \ldots, n+1,
$$

and

$$
D_{i}^{(\infty)}=\left\{\infty<t_{i}<\cdots<t_{n}<x, 0<t_{i-1}<\cdots<t_{1}<1\right\}, \quad i=1, \ldots, n+1,
$$

are expressed in terms of ${ }_{n+1} F_{n}$ in $x$ and in $1 / x$, respectively, under appropriate conditions on the exponents. It is also verified that they provide fundamental sets of solutions of the generalized hypergeometric equation ${ }_{n+1} E_{n}$ around $x=0$ and around $x=\infty$ (see Section 1.1 for the definitions of ${ }_{n+1} F_{n}$ and ${ }_{n+1} E_{n}$ ). Hence, from the viewpoint of twisted de Rham theory, it is expected that the integral (0.1) over an arbitrary twisted cycle $D$ such as

$$
D_{i}=\left\{x<t_{n}<\cdots<t_{i}<0, t_{i}<t_{i-1}<\cdots<t_{1}<1\right\}, \quad i=1, \ldots, n+1,
$$

satisfies the same differential equation ${ }_{n+1} E_{n}$. It is indeed the case as we will prove in Section 6.1.

The contents of this paper are as follows. In Section 1, we recall the classical hypergeometric equations and the hypergeometric system $R E(k, n)$ on the configuration space of $n$ points in the projective $(k-1)$-space. Sections 2-7 are devoted to proving that the integral of a multivalued function for any twisted cycle satisfies the corresponding differential equation(s) in the cases of $E_{A}, E_{B}, E_{C}, E_{D},{ }_{n+1} E_{n}$, and $R E(k, n)$, respectively. As for the cases $E_{D}$ and ${ }_{n+1} E_{n}$, we treat two different types of integrals to help with future studies while in the case of ${ }_{n+1} E_{n}$, each of which can be derived from the other by the change of integration variables.

We note that Yoshida showed in [Y1] that integrals associated with $F_{1}, F_{2}, F_{3}$, and $F_{4}$ satisfy $E_{1}, E_{2}, E_{3}$, and $E_{4}$ for any cycles, and Takano showed in [T] that an integral associated with $F_{C}$ satisfies $E_{C}$ for any cycle. Their arguments, based on the Euler integral transform, are different from ours.

Throughout this paper, we use the symbols

$$
\theta_{x_{i}}=x_{i} \partial / \partial x_{i}, \quad \partial_{x}=\frac{\partial}{\partial x} \quad \text { and } \quad d t=d t_{1} \cdots d t_{n}
$$

for brevity.

\section{Preliminaries}

\subsection{Classical hypergeometric functions}

Lauricella's hypergeometric function $F_{A}$ is the analytic continuation of Lauricella's hypergeometric series

$$
\begin{aligned}
& F_{A}\left(\alpha, \beta_{1}, \ldots, \beta_{n}, \gamma_{1}, \ldots, \gamma_{n} ; x_{1}, \ldots, x_{n}\right) \\
& \quad=\sum_{m_{1}, \ldots, m_{n} \geq 0} \frac{(\alpha)_{m_{1}+\cdots+m_{n}}\left(\beta_{1}\right)_{m_{1}} \cdots\left(\beta_{n}\right)_{m_{n}}}{\left(\gamma_{1}\right)_{m_{1}} \cdots\left(\gamma_{n}\right)_{m_{n}} m_{1} ! \cdots m_{n} !} x_{1}^{m_{1}} \cdots x_{n}^{m_{n}}, \quad\left|x_{1}\right|+\cdots+\left|x_{n}\right|<1,
\end{aligned}
$$


where $(\alpha)_{n}=\alpha(\alpha+1) \cdots(\alpha+n-1)$, and it satisfies the system $E_{A}$ of differential equations of rank $2^{n}$ :

$$
\left(E_{A}\right) \quad \theta_{x_{i}}\left(\theta_{x_{i}}+\gamma_{i}-1\right) F=x_{i}\left(\theta_{x_{i}}+\beta_{i}\right)\left(\theta_{x_{1}}+\cdots+\theta_{x_{n}}+\alpha\right) F, \quad i=1, \ldots, n .
$$

Similarly, Lauricella's hypergeometric functions $F_{B}, F_{C}$, and $F_{D}$ are the analytic continuations of the series

$$
\begin{aligned}
& F_{B}\left(\alpha_{1}, \ldots, \alpha_{n}, \beta_{1}, \ldots, \beta_{n}, \gamma ; x_{1}, \ldots, x_{n}\right) \\
& \quad=\sum_{m_{1}, \ldots, m_{n} \geq 0} \frac{\left(\alpha_{1}\right)_{m_{1}} \cdots\left(\alpha_{n}\right)_{m_{n}}\left(\beta_{1}\right)_{m_{1}} \cdots\left(\beta_{n}\right)_{m_{n}}}{(\gamma)_{m_{1}+\cdots+m_{n}} m_{1} ! \cdots m_{n} !} x_{1}^{m_{1}} \cdots x_{n}^{m_{n}}, \quad\left|x_{1}\right|<1, \ldots,\left|x_{n}\right|<1,
\end{aligned}
$$

$F_{C}\left(\alpha, \beta, \gamma_{1}, \ldots, \gamma_{n} ; x_{1}, \ldots, x_{n}\right)$

$$
=\sum_{m_{1}, \ldots, m_{n} \geq 0} \frac{(\alpha)_{m_{1}+\cdots+m_{n}}(\beta)_{m_{1}+\cdots+m_{n}}}{\left(\gamma_{1}\right)_{m_{1}} \cdots\left(\gamma_{n}\right)_{m_{n}} m_{1} ! \cdots m_{n} !} x_{1}^{m_{1}} \cdots x_{n}^{m_{n}}, \quad \sqrt{\left|x_{1}\right|}+\cdots+\sqrt{\left|x_{n}\right|}<1,
$$

and

$F_{D}\left(\alpha, \beta_{1}, \ldots, \beta_{n}, \gamma ; x_{1}, \ldots, x_{n}\right)$

$$
=\sum_{m_{1}, \ldots, m_{n} \geq 0} \frac{(\alpha)_{m_{1}+\cdots+m_{n}}\left(\beta_{1}\right)_{m_{1}} \cdots\left(\beta_{n}\right)_{m_{n}}}{(\gamma)_{m_{1}+\cdots+m_{n}} m_{1} ! \cdots m_{n} !} x_{1}^{m_{1}} \cdots x_{n}^{m_{n}}, \quad\left|x_{1}\right|<1, \ldots,\left|x_{n}\right|<1,
$$

and they satisfy the following systems $E_{B}, E_{C}$, and $E_{D}$ of rank $2^{n}, 2^{n}$, and $n+1$, respectively:

$$
\begin{gathered}
\left(E_{B}\right) \quad x_{i}\left(\theta_{x_{i}}+\alpha_{i}\right)\left(\theta_{x_{i}}+\beta_{i}\right) F=\theta_{x_{i}}\left(\theta_{x_{1}}+\cdots+\theta_{x_{n}}+\gamma-1\right) F, \quad i=1, \ldots, n, \\
\left(E_{C}\right) \quad \theta_{x_{i}}\left(\theta_{x_{i}}+\gamma_{i}-1\right) F=x_{i}\left(\theta_{x_{1}}+\cdots+\theta_{x_{n}}+\alpha\right)\left(\theta_{x_{1}}+\cdots+\theta_{x_{n}}+\beta\right) F \\
i=1, \ldots, n
\end{gathered}
$$

and

$$
\left(E_{D}\right) \quad\left\{\begin{array}{c}
\theta_{x_{i}}\left(\theta_{x_{1}}+\cdots+\theta_{x_{n}}+\gamma-1\right) F=x_{i}\left(\theta_{x_{i}}+\beta_{i}\right)\left(\theta_{x_{1}}+\cdots+\theta_{x_{n}}+\alpha\right) F, \\
i=1, \ldots, n, \\
x_{i}\left(\theta_{x_{i}}+\beta_{i}\right) \theta_{x_{j}} F=x_{j}\left(\theta_{x_{j}}+\beta_{j}\right) \theta_{x_{i}} F, \quad 1 \leq i \neq j \leq n .
\end{array}\right.
$$

Appell's hypergeometric functions $F_{2}, F_{3}, F_{4}$, and $F_{1}$ are the special $n=2$ cases of $F_{A}, F_{B}$, $F_{C}$, and $F_{D}$, respectively.

The generalized hypergeometric function ${ }_{n+1} F_{n}$ is the analytic continuation of the generalized hypergeometric series

$$
{ }_{n+1} F_{n}\left(\begin{array}{c}
\alpha_{1}, \alpha_{2}, \ldots, \alpha_{n+1} \\
\beta_{1}, \ldots \ldots, \beta_{n}
\end{array} ; x\right)=\sum_{k=0}^{\infty} \frac{\left(\alpha_{1}\right)_{k}\left(\alpha_{2}\right)_{k} \cdots\left(\alpha_{n+1}\right)_{k}}{\left(\beta_{1}\right)_{k} \cdots \cdots\left(\beta_{n}\right)_{k} k !} x^{k}, \quad|x|<1,
$$

and it satisfies the following equation of rank $n+1$ :

$$
\left({ }_{n+1} E_{n}\right) \quad\left[\theta_{x} \prod_{i=1}^{n}\left(\theta_{x}+\beta_{i}-1\right)-x \prod_{i=1}^{n+1}\left(\theta_{x}+\alpha_{i}\right)\right] F=0 .
$$




\subsection{Hypergeometric function on the configuration space}

In this paper, by hypergeometric function $F(k, n)$, we mean the analytic continuation of the series

$F(k, n ; \lambda ; x)$

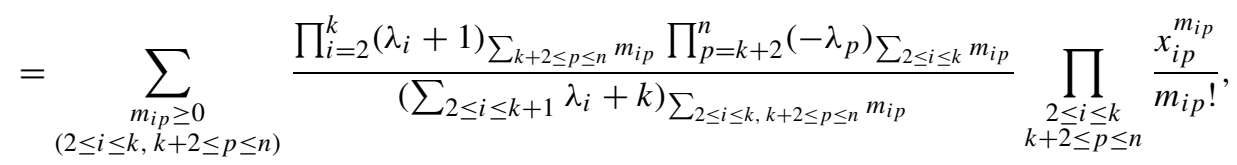

in the variables $x=\left(x_{i p}\right)_{2 \leq i \leq k, k+2 \leq p \leq n}$. It satisfies the following system $R E(k, n)$ of differential equations of rank $\left(\begin{array}{c}n-2 \\ k-1\end{array}\right)$ :

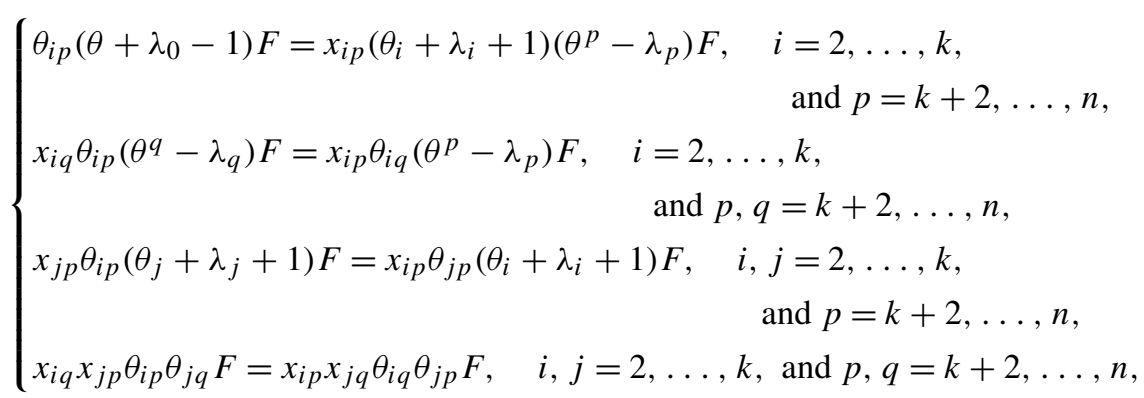

where

$$
\begin{aligned}
\lambda_{0} & =\lambda_{2}+\cdots+\lambda_{k+1}+k, \\
\theta_{j q} & =x_{j q} \frac{\partial}{\partial x_{j q}}, \\
\theta_{j} & =\theta_{j, k+2}+\theta_{j, k+3}+\cdots+\theta_{j, n}, \\
\theta^{q} & =\theta_{2, q}+\theta_{3, q}+\cdots+\theta_{k, q}, \\
\theta & =\theta^{k+2}+\cdots+\theta^{n}=\theta_{2}+\cdots+\theta_{k}=\sum_{\substack{2 \leq i \leq k \\
k+2 \leq r \leq n}} \theta_{i r} .
\end{aligned}
$$

Let $Z_{k, n}$ denote the space of $k \times n$ complex matrices of which all the $k \times k$ minor determinants are non-zero, and let $X_{k, n}:=\mathrm{GL}_{k} \backslash Z_{k, n} / H_{n}$ denote the configuration space of $n$ points in the projective $(k-1)$-space, where $H_{n}$ is the group of diagonal $n \times n$ nonsingular matrices acting on $Z_{k, n}$ by right multiplication. The dimension of $X_{k, n}$ is $(k-1) \times$ $(n-k-1)$, and the variables $x=\left(x_{i p}\right)_{i, p}$ correspond to a system of local coordinates of $X_{k, n}$.

The system of differential equations $R E(k, n)$ on $X_{k, n}$ is called the hypergeometric system on the configuration space of $n$ points in $\mathbb{P}^{k-1}$. This system $R E(k, n)$ is formulated in [MS2] (see also [MSY, Theorem 1.5.1]) and the series $F(k, n)$ can be found, for example, in [MSY, Ki, NN]. We remark that the original system of differential equations $E(k, n)$ due to Gelfand [Ge] is defined on $Z_{k, n}$ and that $R E(k, n)$ is the reduction of $E(k, n)$ to $X_{k, n} ; R E(k, n)$ is sometimes called the reduced system of $E(k, n)$. We refer the reader to [Ge, GKZ, Ho, MSY, MS2, Y2] for more details of these systems. 


\subsection{Twisted homology groups}

Let $u(t)=\prod_{i} f_{i}(t)^{\lambda_{i}}$ be a multivalued function on $T \subset \mathbb{C}^{m}$, where $\lambda_{i} \in \mathbb{C}$ and $T$ is the complement of the singular locus $\bigcup_{i}\left\{f_{i}(t)=0\right\}$ in $\mathbb{C}^{m}$. Let $\mathcal{L}^{\vee}$ be the locally constant sheaf (the local system) defined by $u$ : the sheaf consisting of the local solutions of $d L=L \omega$ for $\omega=d u(t) / u(t)$.

Let $H_{m}\left(T, \mathcal{L}^{\vee}\right)$ be the $m$ th homology group with coefficients in $\mathcal{L}^{\vee}$, and let $H_{m}^{\text {lf }}\left(T, \mathcal{L}^{\vee}\right)$ be the $m$ th locally finite homology group with coefficients in $\mathcal{L}^{\vee}$. Elements of these twisted homology groups, called twisted cycles or loaded cycles, are represented by $\partial$-closed twisted (finite or locally finite) chains

$$
C=\sum_{\Delta} a_{\Delta} \Delta \otimes v_{\Delta} \quad\left(a_{\Delta} \in \mathbb{C}\right),
$$

where each $\Delta$ is an $m$-simplex and $v_{\Delta}$ is a section of $\mathcal{L}^{\vee}$ on $\Delta$.

Under some condition on the exponents $\lambda_{i}$, we have the isomorphism, said to be the regularization,

$$
\text { reg }: H_{m}^{\text {lf }}\left(T, \mathcal{L}^{\vee}\right) \longrightarrow H_{m}\left(T, \mathcal{L}^{\vee}\right),
$$

which is the inverse of the natural map $\iota: H_{m}\left(T, \mathcal{L}^{\vee}\right) \rightarrow H_{m}^{\mathrm{lf}}\left(T, \mathcal{L}^{\vee}\right)$.

For example, in case $T=\mathbb{C} \backslash\{0,1\}$ and $u(t)=t^{\alpha}(t-1)^{\beta}$, where $\alpha, \beta, \alpha+\beta \in \mathbb{R} \backslash \mathbb{Z}$, a regularization (regularized cycle) $\operatorname{reg} C \in H_{1}\left(T, \mathcal{L}^{\vee}\right)$ of $C=\overrightarrow{(0,1)} \in H_{1}^{\mathrm{lf}}\left(T, \mathcal{L}^{\vee}\right)$ can be given by

$$
\operatorname{reg} C=\left\{\frac{1}{d_{\alpha}} S(\epsilon ; 0)+\overrightarrow{[\epsilon, 1-\epsilon]}-\frac{1}{d_{\beta}} S(1-\epsilon ; 1)\right\} \otimes u(t),
$$

where $d_{\lambda}=e^{2 \pi \sqrt{-1} \lambda}-1$, the symbol $S(a ; z)$ stands for the positively oriented circle centered at the point $z$ with starting and ending point $a, \epsilon$ is a small positive number and the argument of each factor of $u(t)$ on the oriented circle $S(\epsilon ; 0)$ or $S(1-\epsilon ; 1)$ is defined so that $\arg t$ takes values from 0 to $2 \pi$ on $S(\epsilon ; 0)$, and $\arg (1-t)$ from 0 to $2 \pi$.

We refer the reader to $[\mathbf{K i}]$ for the construction of regularized cycles in higherdimensional cases.

\section{Lauricella's $\boldsymbol{F}_{\boldsymbol{A}}$}

Let $x=\left(x_{1}, \ldots, x_{n}\right)$ be a point of

$$
X=\mathbb{C}^{n} \backslash \bigcup_{j=1}^{n}\left\{x_{j}=0\right\} \cup \bigcup_{\left\{i_{1}, \ldots, i_{r}\right\} \subset\{1, \ldots, n\}}\left\{\sum_{s=1}^{r} x_{i_{s}}=1\right\} \subset\left(\mathbb{P}^{1}\right)^{n},
$$

and let $u(t)=u(x ; t)$ be a multivalued function

$$
u(x ; t)=\left(1-x_{1} t_{1}-\cdots-x_{n} t_{n}\right)^{-\alpha} \prod_{s=1}^{n} t_{s}^{\beta_{s}-1}\left(1-t_{s}\right)^{\gamma_{s}-\beta_{s}-1}
$$

on

$$
T_{x}=\mathbb{C}^{n} \backslash \bigcup_{j=1}^{n}\left\{t_{j}=0\right\} \cup \bigcup_{j=1}^{n}\left\{t_{j}=1\right\} \cup\left\{1=x_{1} t_{1}+\cdots+x_{n} t_{n}\right\} \subset\left(\mathbb{P}^{1}\right)^{n} .
$$

Let $\mathcal{L}_{x}^{\vee}$ be the locally constant sheaf (local system) determined by $u(t)$.

Then we get Theorem 2.1. 
THEOREM 2.1. We have

$$
\begin{aligned}
& {\left[x_{i}\left(\sum_{s=1}^{n} \theta_{x_{s}}+\alpha\right)\left(\theta_{x_{i}}+\beta_{i}\right)-\theta_{x_{i}}\left(\theta_{x_{i}}+\gamma_{i}-1\right)\right] \int_{C} u(x, t) d t_{1} \cdots d t_{n}=0,} \\
& \quad i=1,2, \ldots, n,
\end{aligned}
$$

for any cycle $C \in H_{n}\left(T_{x}, \mathcal{L}_{x}^{\vee}\right)$.

Proof. Direct calculation shows that

$$
\begin{aligned}
\theta_{x_{i}} u(t) & =\frac{\alpha t_{i} x_{i}}{1-\sum_{1 \leq s \leq n} x_{s} t_{s}} u(t), \\
\theta_{t_{i}} u(t) & =\left[\beta_{i}-1+\frac{\left(\gamma_{i}-\beta_{i}-1\right)\left(-t_{i}\right)}{1-t_{i}}+\frac{\alpha t_{i} x_{i}}{1-\sum_{1 \leq s \leq n} x_{s} t_{s}}\right] u(t) .
\end{aligned}
$$

It follows from (2.1) and (2.2) that

$$
\left(\theta_{x_{i}}-\theta_{t_{i}}\right) u(t)=\left[1-\beta_{i}-\frac{\left(\gamma_{i}-\beta_{i}-1\right)\left(-t_{i}\right)}{1-t_{i}}\right] u(t),
$$

which is equivalent to

$$
\left(\theta_{x_{i}}+\beta_{i}\right) u(t)=\partial_{t_{i}}\left\{t_{i} u(t)\right\}+\frac{\left(\gamma_{i}-\beta_{i}-1\right) t_{i}}{1-t_{i}} u(t),
$$

since $\theta_{t_{i}}=-1+\partial_{t_{i}} t_{i}$, as an operator. Since $t_{i}=1-\left(1-t_{i}\right),(2.3)$ can be written as

$$
\left(\theta_{x_{i}}+\gamma_{i}-1\right) u(t)=\partial_{t_{i}}\left\{t_{i} u(t)\right\}+\left(\gamma_{i}-\beta_{i}-1\right) \frac{1}{1-t_{i}} u(t) .
$$

On the other hand, summing both sides of (2.1) over $i$, we have

$$
\sum_{i=1}^{n} \theta_{x_{i}} u(t)=\frac{\alpha \sum_{i=1}^{n} t_{i} x_{i}}{1-\sum_{1 \leq s \leq n} x_{s} t_{s}} u(t),
$$

which can be written as

$$
\left(\sum_{i=1}^{n} \theta_{x_{i}}+\alpha\right) u(t)=\frac{\alpha}{1-\sum_{1 \leq s \leq n} x_{s} t_{s}} u(t),
$$

since $\sum_{i} t_{i} x_{i}=1-\left(1-\sum_{i} t_{i} x_{i}\right)$.

By (2.1), the action of $\theta_{x_{i}}$ on (2.4) implies

$$
\begin{aligned}
& \theta_{x_{i}}\left(\theta_{x_{i}}+\gamma_{i}-1\right) u(t) \\
& \quad=\partial_{t_{i}}\left\{t_{i} \theta_{x_{i}} u(t)\right\}+\left(\gamma_{i}-\beta_{i}-1\right) \frac{1}{1-t_{i}} \theta_{x_{i}} u(t) \\
& \quad=\partial_{t_{i}}\left\{\frac{\alpha t_{i}^{2} x_{i}}{1-\sum_{1 \leq s \leq n} x_{s} t_{s}} u(t)\right\}+\left(\gamma_{i}-\beta_{i}-1\right) \frac{1}{1-t_{i}} \frac{\alpha t_{i} x_{i}}{1-\sum_{1 \leq s \leq n} x_{s} t_{s}} u(t) .
\end{aligned}
$$


By (2.5), the action of $\left(\sum_{j=1}^{n} \theta_{x_{j}}+\alpha\right)$ on (2.3) implies

$$
\begin{aligned}
& \left(\sum_{j=1}^{n} \theta_{x_{j}}+\alpha\right)\left(\theta_{x_{i}}+\beta_{i}\right) u(t) \\
& \quad=\partial_{t_{i}}\left\{t_{i}\left(\sum_{j=1}^{n} \theta_{x_{j}}+\alpha\right) u(t)\right\}+\frac{\left(\gamma_{i}-\beta_{i}-1\right) t_{i}}{1-t_{i}}\left(\sum_{j=1}^{n} \theta_{x_{j}}+\alpha\right) u(t) \\
& \quad=\partial_{t_{i}}\left\{\frac{\alpha t_{i}}{1-\sum_{1 \leq s \leq n} x_{s} t_{s}} u(t)\right\}+\left(\gamma_{i}-\beta_{i}-1\right) \frac{t_{i}}{1-t_{i}} \frac{\alpha}{1-\sum_{1 \leq s \leq n} x_{s} t_{s}} u(t) .
\end{aligned}
$$

Combining (2.6) and (2.7), we obtain

$$
\left[x_{i}\left(\sum_{s=1}^{n} \theta_{x_{s}}+\alpha\right)\left(\theta_{x_{i}}+\beta_{i}\right)-\theta_{x_{i}}\left(\theta_{x_{i}}+\gamma_{i}-1\right)\right] u(t)=\partial_{t_{i}}\left\{\frac{\alpha x_{i} t_{i}\left(1-t_{i}\right)}{1-\sum_{1 \leq s \leq n} x_{s} t_{s}} u(t)\right\} .
$$

Hence, we reach the result.

Remark 2.2. If we assume the condition for the exponents

$$
\beta_{1}, \ldots, \beta_{n}, \gamma_{1}-\beta_{1}, \ldots, \gamma_{n}-\beta_{n},-\alpha+\sum_{j \in J} \beta_{j} \notin \mathbb{Z}
$$

for all $J \subset\{1, \ldots, n\}$, then the rank of the twisted homology group $H_{n}\left(T_{x}, \mathcal{L}_{x}^{\vee}\right)$ turns out to be $2^{n}$ (see $[\mathbf{A K}, \mathbf{C}, \mathbf{K N}]$ ), and hence it is natural to expect that an appropriate set of $2^{n}$ cycles gives a fundamental set of solutions of $E_{A}$. In the case of $n=2$, it is shown in [M2] by deriving a Wronskian formula that a certain set of four cycles gives a fundamental set of solutions of $E_{A}$, i.e. $E_{2}$.

Remark 2.3. It is shown in [B] that $E_{A}$ is irreducible if and only if (2.8) holds.

Remark 2.4. If $\beta_{j}, \gamma_{j}-\beta_{j} \notin \mathbb{Z}, j=1, \ldots, n$, then we have

$$
\begin{aligned}
& \int_{\operatorname{reg}(0,1)^{n}}\left(1-x_{1} t_{1}-\cdots-x_{n} t_{n}\right)^{-\alpha} \prod_{s=1}^{n} t_{s}^{\beta_{s}-1}\left(1-t_{s}\right)^{\gamma_{s}-\beta_{s}-1} d t_{1} \cdots d t_{n} \\
& \quad=\prod_{j=1}^{n} \frac{\Gamma\left(\beta_{j}\right) \Gamma\left(\gamma_{j}-\beta_{j}\right)}{\Gamma\left(\gamma_{j}\right)} \times F_{A}\left(\alpha, \beta_{1}, \ldots, \beta_{n}, \gamma_{1}, \ldots, \gamma_{n} ; x_{1}, \ldots, x_{n}\right),
\end{aligned}
$$

where the argument of each factor of the integrand is fixed to be zero when $0<x_{1}, 0<$ $x_{2}, \ldots, 0<x_{n}$ and $x_{1}+\cdots+x_{n}<1$; it is the analytic continuation of the integral representation given by Lauricella $[\mathbf{L}]$. See also $[\mathbf{A}]$ and $[\mathbf{A K d F}]$.

\section{Lauricella's $\boldsymbol{F}_{B}$}

Let $x=\left(x_{1}, \ldots, x_{n}\right)$ be a point of

$$
X=\mathbb{C}^{n} \backslash \bigcup_{j=1}^{n}\left\{x_{j}=0\right\} \cup \bigcup_{\left\{i_{1}, \ldots, i_{r}\right\} \subset\{1, \ldots, n\}}\left\{\sum_{s=1}^{r} x_{i_{1}} \cdots \widehat{x_{i_{s}}} \cdots x_{i_{r}}=x_{i_{1}} \cdots x_{i_{r}}\right\} \subset\left(\mathbb{P}^{1}\right)^{n},
$$


where the symbol $\widehat{A}$ means omitting $A$, and let $u(t)=u(x ; t)$ be a multivalued function

$$
u(x ; t)=\left(1-\sum_{s=1}^{n} t_{s}\right)^{\gamma-1-\sum_{1 \leq s \leq n} \alpha_{s}} \prod_{s=1}^{n} t_{s}^{\alpha_{s}-1}\left(1-x_{s} t_{s}\right)^{-\beta_{s}}
$$

on

$$
T_{x}=\mathbb{C}^{n} \backslash \bigcup_{j=1}^{n}\left\{t_{j}=0\right\} \cup \bigcup_{j=1}^{n}\left\{x_{j} t_{j}=1\right\} \cup\left\{t_{1}+\cdots+t_{n}=1\right\} \subset\left(\mathbb{P}^{1}\right)^{n} .
$$

Let $\mathcal{L}_{x}^{\vee}$ be the locally constant sheaf (local system) determined by $u(t)$.

Then we obtain the following.

Theorem 3.1. We have

$$
\left[\theta_{x_{i}}\left(\sum_{s=1}^{n} \theta_{x_{s}}+\gamma-1\right)-x_{i}\left(\theta_{x_{i}}+\alpha_{i}\right)\left(\theta_{x_{i}}+\beta_{i}\right)\right] \int_{C} u(t) d t_{1} \cdots d t_{n}=0, \quad i=1, \ldots, n,
$$

for any cycle $C \in H_{n}\left(T_{x}, \mathcal{L}_{x}^{\vee}\right)$.

Proof. Direct calculation shows that, for $1 \leq i \leq n$,

$$
\begin{aligned}
\theta_{x_{i}} u(t) & =\frac{\beta_{i} t_{i} x_{i}}{1-x_{i} t_{i}} u(t), \\
\theta_{t_{i}} u(t) & =\left[\alpha_{i}-1+\frac{\beta_{i} t_{i} x_{i}}{1-x_{i} t_{i}}+\left(\gamma-1-\sum_{1 \leq s \leq n} \alpha_{s}\right) \frac{-t_{i}}{1-\sum_{1 \leq s \leq n} t_{s}}\right] u(t) .
\end{aligned}
$$

Since $t_{i} x_{i}=1-\left(1-t_{i} x_{i}\right),(3.1)$ can be written as

$$
\left(\theta_{x_{i}}+\beta_{i}\right) u(t)=\frac{\beta_{i}}{1-x_{i} t_{i}} u(t) .
$$

From (3.1) and (3.2), it follows that

$$
\left(\theta_{x_{i}}-\theta_{t_{i}}\right) u(t)=\left[-\alpha_{i}+1+\left(\gamma-1-\sum_{1 \leq s \leq n} \alpha_{s}\right) \frac{t_{i}}{1-\sum_{1 \leq s \leq n} t_{s}}\right] u(t),
$$

which is equivalent to

$$
\left(\theta_{x_{i}}+\alpha_{i}\right) u(t)=\partial_{t_{i}}\left\{t_{i} u(t)\right\}+\left(\gamma-1-\sum_{1 \leq s \leq n} \alpha_{s}\right) \frac{t_{i}}{1-\sum_{1 \leq s \leq n} t_{s}} u(t)
$$

since $\theta_{t_{i}}=-1+\partial_{t_{i}} t_{i}$, as an operator. Summing both sides of (3.4) over $i$, we have

$$
\left(\sum_{1 \leq s \leq n} \theta_{x_{s}}+\gamma-1\right) u(t)=\sum_{1 \leq s \leq n} \partial_{t_{s}}\left\{t_{s} u(t)\right\}+\left(\gamma-1-\sum_{1 \leq s \leq n} \alpha_{s}\right) \frac{1}{1-\sum_{1 \leq s \leq n} t_{s}} u(t)
$$

since $\sum_{s} t_{s}=1-\left(1-\sum_{s} t_{s}\right)$. 
By (3.1), the action of $\theta_{x_{i}}$ on (3.5) implies

$$
\begin{aligned}
& \theta_{x_{i}}\left(\sum_{1 \leq s \leq n} \theta_{x_{s}}+\gamma-1\right) u(t) \\
& \quad=\sum_{1 \leq s \leq m} \partial_{t_{s}}\left\{t_{s} \theta_{x_{i}} u(t)\right\}+\left(\gamma-1-\sum_{1 \leq s \leq n} \alpha_{s}\right) \frac{1}{1-\sum_{1 \leq s \leq n} t_{s}} \theta_{x_{i}} u(t) \\
& \quad=\sum_{1 \leq s \leq n} \partial_{t_{s}}\left\{\frac{\beta_{i} t_{s} t_{i} x_{i}}{1-x_{i} t_{i}} u(t)\right\}+\left(\gamma-1-\sum_{1 \leq s \leq n} a_{s}\right) \frac{1}{1-\sum_{1 \leq s \leq n} t_{s}} \frac{\beta_{i} t_{i} x_{i}}{1-x_{i} t_{i}} u(t) .
\end{aligned}
$$

By (3.3), the action of $\left(\theta_{x_{i}}+\beta_{i}\right)$ on (3.4) implies

$$
\begin{aligned}
& \left(\theta_{x_{i}}+\beta_{i}\right)\left(\theta_{x_{i}}+\alpha_{i}\right) u(t) \\
& \quad=\partial_{t_{i}}\left\{t_{i}\left(\theta_{x_{i}}+\beta_{i}\right) u(t)\right\}+\left(\gamma-1-\sum_{1 \leq s \leq n} \alpha_{s}\right) \frac{t_{i}}{1-\sum_{1 \leq s \leq n} t_{s}}\left(\theta_{x_{i}}+\beta_{i}\right) u(t) \\
& \quad=\partial_{t_{i}}\left\{\frac{\beta_{i} t_{i}}{1-x_{i} t_{i}} u(t)\right\}+\left(\gamma-1-\sum_{1 \leq s \leq n} \alpha_{s}\right) \frac{t_{i}}{1-\sum_{1 \leq s \leq n} t_{s}} \frac{\beta_{i}}{1-x_{i} t_{i}} u(t) .
\end{aligned}
$$

Combining (3.6) and (3.7), we obtain

$$
\begin{gathered}
{\left[\theta_{x_{i}}\left(\sum_{s=1}^{n} \theta_{x_{s}}+\gamma-1\right)-x_{i}\left(\theta_{x_{i}}+\alpha_{i}\right)\left(\theta_{x_{i}}+\beta_{i}\right)\right] u(t)} \\
=\sum_{1 \leq s \leq n} \partial_{t_{s}}\left\{\frac{\beta_{i} x_{i} t_{i} t_{s}}{1-x_{i} t_{i}} u(t)\right\}-\partial_{t_{i}}\left\{\frac{\beta_{i} x_{i} t_{i}}{1-x_{i} t_{i}} u(t)\right\} .
\end{gathered}
$$

Therefore, we arrive at the result.

Remark 3.2. If we assume the condition for the exponents

$$
\alpha_{1}, \ldots, \alpha_{n}, \beta_{1}, \ldots, \beta_{n}, \gamma-\delta_{1}-\cdots-\delta_{n} \notin \mathbb{Z},
$$

for $\delta_{i} \in\left\{\alpha_{i}, \beta_{i}\right\}$, then the rank of $H_{n}\left(T_{x}, \mathcal{L}_{x}^{\vee}\right)$ is $2^{n}$, and an appropriate set of $2^{n}$ cycles would give rise to a fundamental set of solutions of $E_{B}$. In the case of $n=2$, it is shown in [M2] by deriving a Wronskian formula that a certain set of four cycles gives a fundamental set of solutions of $E_{B}$, i.e. $E_{3}$.

Remark 3.3. It is shown in [B] that $E_{B}$ is irreducible if and only if (3.8) holds.

Remark 3.4. If $\alpha_{1}, \ldots, \alpha_{n}, \gamma-\alpha_{1}-\cdots-\alpha_{n} \notin \mathbb{Z}$, then we have

$$
\begin{aligned}
& \int_{\operatorname{reg}\left(t_{1}>0, \ldots, t_{n}>0,1>t_{1}+\cdots+t_{n}\right)} \prod_{s=1}^{n}\left\{t_{s}^{\alpha_{s}-1}\left(1-x_{s} t_{s}\right)^{-\beta_{s}}\right\}\left(1-\sum_{s=1}^{n} t_{s}\right)^{\gamma-1-\sum_{1 \leq s \leq n} \alpha_{s}} d t_{1} \cdots d t_{n} \\
& =\frac{\Gamma\left(\alpha_{1}\right) \cdots \Gamma\left(\alpha_{n}\right) \Gamma\left(\gamma-\alpha_{1}-\cdots-\alpha_{n}\right)}{\Gamma(\gamma)} \\
& \quad \times F_{B}\left(\alpha_{1}, \ldots, \alpha_{n}, \beta_{1}, \ldots, \beta_{n}, \gamma ; x_{1}, \ldots, x_{n}\right),
\end{aligned}
$$

where the argument of each factor of the integrand is fixed to be zero when $0<x_{i}<1, i=$ $1, \ldots, n$; it is the analytic continuation of the integral representation given by Lauricella [L]. See also $[\mathbf{A K d F}]$. 


\section{Lauricella's $F_{C}$}

Let $x=\left(x_{1}, \ldots, x_{n}\right)$ be a point of

$$
X=\mathbb{C}^{n} \backslash \bigcup_{s=1}^{n}\left\{x_{s}=0\right\} \cup \bigcup_{\epsilon_{i} \in\{-1,1\}}\left\{1+\epsilon_{1} \sqrt{x_{1}}+\cdots+\epsilon_{n} \sqrt{x_{n}}=0\right\} \subset\left(\mathbb{P}^{1}\right)^{n},
$$

and let $u(t)=u(x ; t)$ be a multivalued function

$$
u(x ; t)=\left(1-\sum_{s=1}^{n} t_{s}\right)^{-\alpha-n+\sum_{1 \leq s \leq n} \gamma_{s}}\left\{\prod_{s=1}^{n} t_{s}^{-\gamma_{s}}\right\}\left(1-\sum_{s=1}^{n} \frac{x_{s}}{t_{s}}\right)^{-\beta}
$$

on

$$
T_{x}=\mathbb{C}^{n} \backslash \bigcup_{s=1}^{n}\left\{t_{s}=0\right\} \cup\left\{\sum_{s=1}^{n} x_{s} t_{1} \cdots \widehat{t_{s}} \cdots t_{n}=t_{1} \cdots t_{n}\right\} \cup\left\{\sum_{s=1}^{n} t_{s}=1\right\} \subset\left(\mathbb{P}^{1}\right)^{n} .
$$

Let $\mathcal{L}_{x}^{\vee}$ be the locally constant sheaf (local system) determined by $u(t)$.

Then we obtain the following.

THEOREM 4.1. We have

$$
\begin{aligned}
& {\left[\theta_{x_{i}}\left(\theta_{x_{i}}+\gamma_{i}-1\right)-x_{i}\left(\sum_{1 \leq s \leq n} \theta_{x_{s}}+\beta\right)\left(\sum_{1 \leq s \leq n} \theta_{x_{s}}+\alpha\right)\right]} \\
& \times \int_{C} u(t) d t_{1} \cdots d t_{n}=0, \quad i=1,2, \ldots, n,
\end{aligned}
$$

for any cycle $C \in H_{n}\left(T_{x}, \mathcal{L}_{x}^{\vee}\right)$.

Proof. Direct calculation shows that

$$
\begin{aligned}
\theta_{x_{i}} u(t) & =\frac{\beta\left(x_{i} / t_{i}\right)}{1-\sum_{s=1}^{n}\left(x_{s} / t_{s}\right)} u(t), \\
\theta_{t_{i}} u(t) & =\left[-\gamma_{i}+\frac{-\beta\left(x_{i} / t_{i}\right)}{1-\sum_{s=1}^{n}\left(x_{s} / t_{s}\right)}+\left(\sum_{1 \leq s \leq n} \gamma_{s}-\alpha-n\right) \frac{-t_{i}}{1-\sum_{1 \leq s \leq n} t_{s}}\right] u(t) .
\end{aligned}
$$

Summing both sides of (4.1) over $i$, we have

$$
\left(\sum_{1 \leq s \leq n} \theta_{x_{s}}+\beta\right) u(t)=\frac{\beta}{1-\sum_{s=1}^{n}\left(x_{s} / t_{s}\right)} u(t),
$$

since $\sum_{s}\left(x_{s} / t_{s}\right)=1-\left(1-\sum_{s}\left(x_{s} / t_{S}\right)\right)$. From (4.1) and (4.2), it follows that

$$
\left(\theta_{x_{i}}+\theta_{t_{i}}+\gamma_{i}\right) u(t)=\left(-\alpha-n+\sum_{1 \leq s \leq n} \gamma_{s}\right) \frac{-t_{i}}{1-\sum_{1 \leq s \leq n} t_{s}} u(t),
$$

which is equivalent to

$$
\left(\theta_{x_{i}}-1+\gamma_{i}\right) u(t)=-\partial_{t_{i}}\left\{t_{i} u(t)\right\}+\left(\alpha+n-\sum_{1 \leq s \leq n} \gamma_{s}\right) \frac{t_{i}}{1-\sum_{1 \leq s \leq n} t_{s}} u(t)
$$

since $\theta_{t_{i}}=-1+\partial_{t_{i}} t_{i}$, as an operator. 
On the other hand, summing both sides of (4.4) over $i$, we have

$$
\left(\sum_{1 \leq s \leq n} \theta_{x_{s}}+\alpha\right) u(t)=-\sum_{1 \leq s \leq n} \partial_{t_{s}}\left\{t_{s} u(t)\right\}+\left(\alpha+n-\sum_{1 \leq s \leq n} \gamma_{s}\right) \frac{1}{1-\sum_{1 \leq s \leq n} t_{s}} u(t),
$$

since $-\sum_{s} t_{s}=-1+\left(1-\sum_{s} t_{s}\right)$.

By (4.1), the action of $\theta_{x_{i}}$ on (4.4) implies

$$
\begin{aligned}
\theta_{x_{i}}\left(\theta_{x_{i}}-1+\gamma_{i}\right) u(t)= & -\partial_{t_{i}}\left\{t_{i} \theta_{x_{i}} u(t)\right\}+\left(\alpha+n-\sum_{1 \leq s \leq n} \gamma_{s}\right) \frac{t_{i}}{1-\sum_{1 \leq s \leq n} t_{s}} \theta_{x_{i}} u(t) \\
= & -\partial_{t_{i}}\left\{\frac{\beta x_{i}}{1-\sum_{s=1}^{n}\left(x_{s} / t_{s}\right)} u(t)\right\} \\
& +\left(\alpha+n-\sum_{1 \leq s \leq n} \gamma_{s}\right) \frac{1}{1-\sum_{1 \leq s \leq n} t_{s}} \frac{\beta x_{i}}{1-\sum_{s=1}^{n}\left(x_{s} / t_{s}\right)} u(t) .
\end{aligned}
$$

By (4.3), the action of $\left(\sum_{1 \leq s \leq n} \theta_{x_{s}}+\beta\right)$ on (4.5) implies

$$
\begin{aligned}
\left(\sum_{1 \leq s \leq n} \theta_{x_{s}}+\beta\right)\left(\sum_{1 \leq s \leq n} \theta_{x_{s}}+\alpha\right) u(t) \\
=-\sum_{1 \leq s \leq n} \partial_{t_{s}}\left\{t_{s}\left(\sum_{1 \leq s \leq n} \theta_{x_{s}}+\beta\right) u(t)\right\} \\
\quad+\left(\alpha+n-\sum_{1 \leq s \leq n} \gamma_{s}\right) \frac{1}{1-\sum_{1 \leq s \leq n} t_{s}}\left(\sum_{1 \leq s \leq n} \theta_{x_{s}}+\beta\right) u(t) \\
=-\sum_{1 \leq s \leq n} \partial_{t_{s}}\left\{\frac{\beta t_{s}}{1-\sum_{s=1}^{n}\left(x_{s} / t_{s}\right)} u(t)\right\}+\left(\alpha+n-\sum_{1 \leq s \leq n} \gamma_{s}\right) \frac{\beta}{1-\sum_{s=1}^{n}\left(x_{s} / t_{s}\right)} u(t) .
\end{aligned}
$$

Combining (4.6) and (4.7), we obtain

$$
\begin{aligned}
& {\left[\theta_{x_{i}}\left(\theta_{x_{i}}+\gamma_{i}-1\right)-x_{i}\left(\sum_{1 \leq s \leq n} \theta_{x_{s}}+\beta\right)\left(\sum_{1 \leq s \leq n} \theta_{x_{s}}+\alpha\right)\right] u(t)} \\
& \quad=\sum_{1 \leq s \leq n} \partial_{t_{s}}\left\{\frac{\beta x_{i} t_{s}}{1-\sum_{s=1}^{n}\left(x_{s} / t_{s}\right)} u(t)\right\}-\partial_{t_{i}}\left\{\frac{\beta x_{i}}{1-\sum_{s=1}^{n}\left(x_{s} / t_{s}\right)} u(t)\right\} .
\end{aligned}
$$

Therefore, we arrive at the result.

Remark 4.2. If we assume the condition for the exponents

$$
-\alpha+\sum_{i \in I} \gamma_{i},-\beta+\sum_{i \in I} \gamma_{i} \notin \mathbb{Z}
$$

for all $I \subset\{1, \ldots, n\}$, then the rank of $H_{n}\left(T_{x}, \mathcal{L}_{x}^{\vee}\right)$ is $2^{n}$, and a set of $2^{n}$ cycles might give rise to a fundamental set of solutions of $E_{C}$.

Remark 4.3. It is shown in [B] that $E_{C}$ is irreducible if and only if (4.8) holds. 
Remark 4.4. If $\gamma_{1}, \ldots, \gamma_{n}, \gamma_{1}+\cdots+\gamma_{n}-\alpha \notin \mathbb{Z}$, then we have

$$
\begin{aligned}
& \int_{\operatorname{reg}\left(t_{1}>0, \ldots, t_{n}>0,1>t_{1}+\cdots+t_{n}\right)}\left\{\prod_{s=1}^{n} t_{s}^{-\gamma_{s}}\right\} \\
& \quad \times\left(1-\sum_{s=1}^{n} t_{s}\right)^{-\alpha-n+\sum_{1 \leq s \leq n} \gamma_{s}}\left(1-\sum_{s=1}^{n} \frac{x_{s}}{t_{s}}\right)^{-\beta} d t_{1} \cdots d t_{n} \\
& =\frac{\Gamma\left(1-\gamma_{1}\right) \cdots \Gamma\left(1-\gamma_{n}\right) \Gamma\left(\gamma_{1}+\cdots+\gamma_{n}+1-n-\alpha\right)}{\Gamma(1-\alpha)} \\
& \quad \times F_{C}\left(\alpha, \beta, \gamma_{1}, \ldots, \gamma_{n} ; x_{1}, \ldots, x_{n}\right),
\end{aligned}
$$

where the argument of each factor of the integrand is fixed to be zero when $x_{1}>0, \ldots, x_{n}>$ 0 are small. This formula was established by Aomoto and Kita $[\mathbf{A K}]$ and Kita [Ki]. See also Proposition 2 of [Ka], and [Pa]. In contrast to the other classical hypergeometric functions, the regularization of the cycle is essential in this integral representation. It is impossible to define the integral on the open set $\left\{t_{1}>0, \ldots, t_{n}>0,1>t_{1}+\cdots+t_{n}\right\}$ even in the case where the real part of the exponents are positive.

\section{Lauricella's $\boldsymbol{F}_{D}$}

\subsection{Integrals of one variable}

Let $x=\left(x_{1}, \ldots, x_{n}\right)$ be a point of

$$
X=\mathbb{C}^{n} \backslash \bigcup_{j=1}^{n}\left\{x_{j}=0\right\} \cup \bigcup_{j=1}^{n}\left\{x_{j}=1\right\} \cup \bigcup_{1 \leq i<j \leq n}\left\{x_{i}=x_{j}\right\} \subset\left(\mathbb{P}^{1}\right)^{n},
$$

and let $u(t)=u(x ; t)$ be a multivalued function

$$
u(x ; t)=t^{\lambda_{0}} \prod_{j=1}^{n+1}\left(t-x_{j}\right)^{\lambda_{j}} \quad \text { with } x_{n+1}=1,
$$

on

$$
T_{x}=\mathbb{C} \backslash\left\{0,1, x_{1}, \ldots, x_{n}\right\} \subset \mathbb{P}^{1},
$$

where

$$
\lambda_{0}=\beta_{1}+\cdots+\beta_{n}-\gamma, \quad \lambda_{j}=-\beta_{j}(1 \leq j \leq n), \quad \lambda_{n+1}=\gamma-\alpha-1 .
$$

Let $\mathcal{L}_{x}^{\vee}$ be the locally constant sheaf (local system) determined by $u(t)$.

Then we get Theorem 5.1.

THEOREM 5.1. We have

$$
\begin{array}{r}
{\left[\theta_{x_{i}}\left(\sum_{s=1}^{n} \theta_{x_{s}}+\gamma-1\right)-x_{i}\left(\theta_{x_{i}}+\beta_{i}\right)\left(\sum_{s=1}^{n} \theta_{x_{s}}+\alpha\right)\right] \int_{C} u(t) d t=0, \quad i=1, \ldots, n,} \\
{\left[x_{i}\left(\theta_{x_{i}}+\beta_{i}\right) \theta_{x_{j}}-x_{j}\left(\theta_{x_{j}}+\beta_{j}\right) \theta_{x_{i}}\right] \int_{C} u(t) d t=0, \quad 1 \leq i \neq j \leq n,}
\end{array}
$$

for any cycle $C \in H_{1}\left(T_{x}, \mathcal{L}_{x}^{\vee}\right)$. 
Proof. Direct calculation shows that

$$
\begin{aligned}
\theta_{x_{i}} u(t) & =\frac{-\lambda_{i} x_{i}}{t-x_{i}} u(t), \\
\theta_{t} u(t) & =\left[\lambda_{0}+\sum_{1 \leq j \leq n} \frac{\lambda_{j} t}{t-x_{j}}+\frac{\lambda_{n+1} t}{t-1}\right] u(t) .
\end{aligned}
$$

Since $-x_{i}=\left(t-x_{i}\right)-t,(5.2)$ implies

$$
\left(\theta_{x_{i}}-\lambda_{i}\right) u(t)=\frac{-\lambda_{i} t}{t-x_{i}} u(t)
$$

Summing both sides of (5.2) over $i$ and both sides of (5.3), we have

$$
\left(\sum_{1 \leq s \leq n} \theta_{x_{s}}+\theta_{t}\right) u(t)=\left(\sum_{0 \leq j \leq n} \lambda_{j}+\frac{\lambda_{n+1} t}{t-1}\right) u(t),
$$

which is equivalent to

$$
\left(\sum_{1 \leq s \leq n} \theta_{x_{s}}-1-\sum_{0 \leq j \leq n} \lambda_{j}\right) u(t)=-\partial_{t}\{t u(t)\}+\frac{\lambda_{n+1} t}{t-1} u(t),
$$

since $\theta_{t_{i}}=-1+\partial_{t_{i}} t_{i}$, as an operator. Since $t=1+(t-1),(5.5)$ can be written as

$$
\left(\sum_{1 \leq s \leq n} \theta_{x_{s}}-1-\sum_{0 \leq j \leq n+1} \lambda_{j}\right) u(t)=-\partial_{t}\{t u(t)\}+\frac{\lambda_{n+1}}{t-1} u(t) .
$$

By (5.2), the action of $\theta_{x_{i}}$ on (5.5) implies

$$
\begin{aligned}
\theta_{x_{i}}\left(\sum_{1 \leq s \leq n} \theta_{x_{s}}-1-\sum_{0 \leq j \leq n} \lambda_{j}\right) u(t) & =-\partial_{t}\left\{t \theta_{x_{i}} u(t)\right\}+\frac{\lambda_{n+1} t}{t-1} \theta_{x_{i}} u(t) \\
& =-\partial_{t}\left\{t \frac{-\lambda_{i} x_{i}}{t-x_{i}} u(t)\right\}+\frac{\lambda_{n+1} t}{t-1} \frac{-\lambda_{i} x_{i}}{t-x_{i}} u(t)
\end{aligned}
$$

By (5.4), the action of $\left(\theta_{x_{i}}-\lambda_{i}\right)$ on (5.6) implies

$$
\begin{aligned}
\left(\theta_{x_{i}}-\lambda_{i}\right)\left(\sum_{1 \leq s \leq n} \theta_{x_{s}}-1-\sum_{0 \leq j \leq n+1} \lambda_{j}\right) u(t) & =-\partial_{t}\left\{t\left(\theta_{x_{i}}-\lambda_{i}\right) u(t)\right\}+\frac{\lambda_{n+1}}{t-1}\left(\theta_{x_{i}}-\lambda_{i}\right) u(t) \\
& =-\partial_{t}\left\{t \frac{-\lambda_{i} t}{t-x_{i}} u(t)\right\}+\frac{\lambda_{n+1}}{t-1} \frac{-\lambda_{i} t}{t-x_{i}} u(t) .
\end{aligned}
$$

Combining (5.7) and (5.8), we obtain

$$
\begin{aligned}
& {\left[x_{i}\left(\theta_{x_{i}}-\lambda_{i}\right)\left(\sum_{1 \leq s \leq n} \theta_{x_{s}}-1-\sum_{0 \leq j \leq n+1} \lambda_{j}\right)-\theta_{x_{i}}\left(\sum_{1 \leq s \leq n} \theta_{x_{s}}-1-\sum_{0 \leq j \leq n} \lambda_{j}\right)\right] u(t)} \\
& \quad=\lambda_{i} x_{i} \partial_{t}\left\{\frac{t(t-1)}{t-x_{i}} u(t)\right\},
\end{aligned}
$$


which leads to

$$
\begin{aligned}
& {\left[x_{i}\left(\theta_{x_{i}}-\lambda_{i}\right)\left(\sum_{1 \leq s \leq n} \theta_{x_{s}}-1-\sum_{0 \leq j \leq n+1} \lambda_{j}\right)\right.} \\
& \left.\quad-\theta_{x_{i}}\left(\sum_{1 \leq s \leq n} \theta_{x_{s}}-1-\sum_{0 \leq j \leq n} \lambda_{j}\right)\right] \int_{C} u(t) d t=0, \quad i=1, \ldots, n,
\end{aligned}
$$

for a cycle $C$.

On the other hand, combining (5.2) and (5.4), we have

$$
\begin{aligned}
x_{i} \theta_{x_{j}}\left(\theta_{x_{i}}-\lambda_{i}\right) u(t) & =x_{i} \theta_{x_{j}} \frac{-\lambda_{i} t}{t-x_{i}} u(t) \\
& =x_{i} \frac{-\lambda_{i} t}{t-x_{i}} \frac{-\lambda_{j} x_{j}}{t-x_{j}} u(t)=\frac{\lambda_{i} \lambda_{j} x_{i} x_{j} t}{\left(t-x_{i}\right)\left(t-x_{j}\right)} u(t),
\end{aligned}
$$

and

$$
\begin{aligned}
x_{j} \theta_{x_{i}}\left(\theta_{x_{j}}-\lambda_{j}\right) u(t) & =x_{j} \theta_{x_{i}} \frac{-\lambda_{j} t}{t-x_{j}} u(t) \\
& =x_{j} \frac{-\lambda_{j} t}{t-x_{j}} \frac{-\lambda_{i} x_{i}}{t-x_{i}} u(t)=\frac{\lambda_{i} \lambda_{j} x_{i} x_{j} t}{\left(t-x_{i}\right)\left(t-x_{j}\right)} u(t),
\end{aligned}
$$

which lead to

$$
\left[x_{i} \theta_{x_{j}}\left(\theta_{x_{i}}-\lambda_{i}\right)-x_{j} \theta_{x_{i}}\left(\theta_{x_{j}}-\lambda_{j}\right)\right] u(t)=0, \quad 1 \leq i \neq j \leq n,
$$

and thus

$$
\left[x_{i} \theta_{x_{j}}\left(\theta_{x_{i}}-\lambda_{i}\right)-x_{j} \theta_{x_{i}}\left(\theta_{x_{j}}-\lambda_{j}\right)\right] \int_{C} u(t) d t=0, \quad 1 \leq i \neq j \leq n,
$$

for a cycle $C$.

Substitution of (5.1) implies the result.

Remark 5.2. If we assume the condition for the exponents

$$
\alpha, \beta_{1}, \ldots, \beta_{n}, \gamma-\alpha, \gamma-\beta_{1}-\cdots-\beta_{n} \notin \mathbb{Z},
$$

then the rank of $H_{1}\left(T_{x}, \mathcal{L}_{x}^{\vee}\right)$ is $n+1$, and it is proved in [MS1] that a set of $n+1$ cycles give rise to a fundamental set of solutions of $E_{D}$.

Remark 5.3. It is shown in [B] and [MS1] that $E_{D}$ is irreducible if and only if (5.9) holds.

Remark 5.4. If $\alpha, \gamma-\alpha \notin \mathbb{Z}$, then we have

$$
\begin{aligned}
& \int_{\operatorname{reg}(1, \infty)} t^{\beta_{1}+\cdots+\beta_{n}-\gamma}\left(t-x_{1}\right)^{-\beta_{1}} \cdots\left(t-x_{n}\right)^{-\beta_{n}}(t-1)^{\gamma-\alpha-1} d t \\
& \quad=\frac{\Gamma(\alpha) \Gamma(\gamma-\alpha)}{\Gamma(\gamma)} \times F_{D}\left(\alpha, \beta_{1}, \ldots, \beta_{n}, \gamma ; x_{1}, \ldots, x_{n}\right)
\end{aligned}
$$

where the argument of each factor of the integrand is fixed to be zero when $x_{1}, \ldots, x_{n}<1$; this is a modification of the formula by Lauricella [L]. See also [MS1]. 


\subsection{Integrals of many variables}

Let $x=\left(x_{1}, \ldots, x_{n}\right)$ be a point of

$$
X=\mathbb{C}^{n} \backslash \bigcup_{j=1}^{n}\left\{x_{j}=0\right\} \cup \bigcup_{j=1}^{n}\left\{x_{j}=1\right\} \cup \bigcup_{1 \leq i<j \leq n}\left\{x_{i}=x_{j}\right\} \subset\left(\mathbb{P}^{1}\right)^{n},
$$

and let $u(t)=u(x ; t)$ be a multivalued function

$$
u(x ; t)=\left(1-\sum_{s=1}^{n} t_{s}\right)^{\lambda_{0}}\left(1-\sum_{s=1}^{n} t_{s} x_{s}\right)^{-\alpha} \prod_{s=1}^{n} t_{s}^{\beta_{s}-1},
$$

on

$$
T_{x}=\mathbb{C} \backslash \bigcup_{j=1}^{n}\left\{t_{j}=0\right\} \cup\left\{\sum_{s=1}^{n} t_{s}=1\right\} \cup\left\{\sum_{s=1}^{n} t_{s} x_{s}=1\right\} \subset \mathbb{P}^{n}
$$

where

$$
\lambda_{0}=\gamma-1-\beta_{1}-\cdots-\beta_{n} .
$$

Let $\mathcal{L}_{x}^{\vee}$ be the locally constant sheaf (local system) determined by $u(t)$.

Then we get the following.

THEOREM 5.5. We have

$$
\begin{array}{r}
{\left[\theta_{x_{i}}\left(\sum_{s=1}^{n} \theta_{x_{s}}+\gamma-1\right)-x_{i}\left(\theta_{x_{i}}+\beta_{i}\right)\left(\sum_{s=1}^{n} \theta_{x_{s}}+\alpha\right)\right] \int_{C} u(t) d t=0, \quad i=1, \ldots, n,} \\
{\left[x_{i}\left(\theta_{x_{i}}+\beta_{i}\right) \theta_{x_{j}}-x_{j}\left(\theta_{x_{j}}+\beta_{j}\right) \theta_{x_{i}}\right] \int_{C} u(t) d t=0, \quad 1 \leq i \neq j \leq n,}
\end{array}
$$

for any cycle $C \in H_{n}\left(T_{x}, \mathcal{L}_{x}^{\vee}\right)$.

Proof. Direct calculation shows that

$$
\begin{aligned}
\theta_{x_{i}} u(t) & =\frac{\alpha t_{i} x_{i}}{1-\sum_{1 \leq s \leq n} x_{s} t_{s}} u(t), \\
\theta_{t_{i}} u(t) & =\left[\beta_{i}-1+\frac{-\lambda_{0} t_{i}}{1-\sum_{1 \leq s \leq n} t_{s}}+\frac{\alpha t_{i} x_{i}}{1-\sum_{1 \leq s \leq n} x_{s} t_{s}}\right] u(t) .
\end{aligned}
$$

It follows from (5.11) and (5.12) that

$$
\left(\theta_{x_{i}}-\theta_{t_{i}}\right) u(t)=\left[1-\beta_{i}+\frac{\lambda_{0} t_{i}}{1-\sum_{1 \leq s \leq n} t_{s}}\right] u(t),
$$

which is equivalent to

$$
\left(\theta_{x_{i}}+\beta_{i}\right) u(t)=\partial_{t_{i}}\left\{t_{i} u(t)\right\}+\frac{\lambda_{0} t_{i}}{1-\sum_{1 \leq s \leq n} t_{s}} u(t),
$$

since $\theta_{t_{i}}=-1+\partial_{t_{i}} t_{i}$, as an operator. Moreover, summing both sides of (5.13) over $i$, we have

$$
\left(\sum_{i=1}^{n}\left(\theta_{x_{i}}+\beta_{i}\right)\right) u(t)=\sum_{i=1}^{n} \partial_{t_{i}}\left\{t_{i} u(t)\right\}+\frac{\lambda_{0} \sum_{i=1}^{n} t_{i}}{1-\sum_{1 \leq s \leq n} t_{s}} u(t),
$$


which can be written as

$$
\left(\sum_{i=1}^{n} \theta_{x_{i}}+\gamma-1\right) u(t)=\sum_{i=1}^{n} \partial_{t_{i}}\left\{t_{i} u(t)\right\}+\frac{\lambda_{0}}{1-\sum_{1 \leq s \leq n} t_{s}} u(t),
$$

since $\sum_{i=1}^{n} t_{i}=1-\left(1-\sum_{i=1}^{n} t_{i}\right)$ and (5.10).

On the other hand, summing both sides of (5.11) over $i$, we have

$$
\sum_{i=1}^{n} \theta_{x_{i}} u(t)=\frac{\alpha \sum_{i=1}^{n} t_{i} x_{i}}{1-\sum_{1 \leq s \leq n} x_{s} t_{s}} u(t),
$$

which can be written as

$$
\left(\sum_{i=1}^{n} \theta_{x_{i}}+\alpha\right) u(t)=\frac{\alpha}{1-\sum_{1 \leq s \leq n} x_{s} t_{s}} u(t),
$$

since $\sum_{i} t_{i} x_{i}=1-\left(1-\sum_{i} t_{i} x_{i}\right)$.

By (5.11), the action of $\theta_{x_{i}}$ on (5.14) implies

$$
\begin{aligned}
\theta_{x_{i}}\left(\sum_{s=1}^{n} \theta_{x_{s}}+\gamma-1\right) u(t)= & \sum_{s=1}^{n} \partial_{t_{s}}\left\{t_{s} \theta_{x_{i}} u(t)\right\}+\frac{\lambda_{0}}{1-\sum_{1 \leq s \leq n} t_{s}} \theta_{x_{i}} u(t) \\
= & \sum_{s=1}^{n} \partial_{t_{s}}\left\{t_{s} \frac{\alpha t_{i} x_{i}}{1-\sum_{1 \leq s \leq n} x_{s} t_{s}} u(t)\right\} \\
& +\frac{\lambda_{0}}{1-\sum_{1 \leq s \leq n} t_{s}} \frac{\alpha t_{i} x_{i}}{1-\sum_{1 \leq s \leq n} x_{s} t_{s}} u(t) .
\end{aligned}
$$

By (5.15), the action of $\left(\sum_{s=1}^{n} \theta_{x_{s}}+\alpha\right)$ on (5.13) implies

$$
\begin{aligned}
& \left(\sum_{s=1}^{n} \theta_{x_{s}}+\alpha\right)\left(\theta_{x_{i}}+\beta_{i}\right) u(t) \\
& \quad=\partial_{t_{i}}\left\{t_{i}\left(\sum_{s=1}^{n} \theta_{x_{s}}+\alpha\right) u(t)\right\}+\frac{\lambda_{0} t_{i}}{1-\sum_{1 \leq s \leq n} x_{s} t_{s}}\left(\sum_{s=1}^{n} \theta_{x_{s}}+\alpha\right) u(t) \\
& \quad=\partial_{t_{i}}\left\{t_{i} \frac{\alpha}{1-\sum_{1 \leq s \leq n} x_{s} t_{s}} u(t)\right\}+\frac{\lambda_{0} t_{i}}{1-\sum_{1 \leq s \leq n} x_{s} t_{s}} \frac{\alpha}{1-\sum_{1 \leq s \leq n} x_{s} t_{s}} u(t) .
\end{aligned}
$$

Combining (5.16) and (5.17), we obtain

$$
\begin{aligned}
& {\left[x_{i}\left(\sum_{s=1}^{n} \theta_{x_{s}}+\alpha\right)\left(\theta_{x_{i}}+\beta_{i}\right)-\theta_{x_{i}}\left(\sum_{s=1}^{n} \theta_{x_{s}}+\gamma-1\right)\right] u(t)} \\
& \quad=x_{i} \partial_{t_{i}}\left\{t_{i} \frac{\alpha}{1-\sum_{1 \leq s \leq n} x_{s} t_{s}} u(t)\right\}-\sum_{s=1}^{n} \partial_{t_{s}}\left\{t_{s} \frac{\alpha t_{i} x_{i}}{1-\sum_{1 \leq s \leq n} x_{s} t_{s}} u(t)\right\},
\end{aligned}
$$

which leads to

$$
\left[x_{i}\left(\sum_{s=1}^{n} \theta_{x_{s}}+\alpha\right)\left(\theta_{x_{i}}+\beta_{i}\right)-\theta_{x_{i}}\left(\sum_{s=1}^{n} \theta_{x_{s}}+\gamma-1\right)\right] \int_{C} u(t) d t=0, \quad i=1, \ldots, n,
$$

for any cycle $C$. 
On the other hand, combining (5.11) and (5.13), we have

$$
\begin{aligned}
x_{i} \theta_{x_{j}}\left(\theta_{x_{i}}+\beta_{i}\right) u(t) & =x_{i} \partial_{t_{i}}\left\{t_{i} \theta_{x_{j}} u(t)\right\}+x_{i} \frac{\lambda_{0} t_{i}}{1-\sum_{1 \leq s \leq n} t_{s}} \theta_{x_{j}} u(t) \\
& =x_{i} \partial_{t_{i}}\left\{t_{i} \theta_{x_{j}} u(t)\right\}+x_{i} \frac{\lambda_{0} t_{i}}{1-\sum_{1 \leq s \leq n} t_{s}} \frac{\alpha t_{j} x_{j}}{1-\sum_{1 \leq s \leq n} x_{s} t_{s}} u(t)
\end{aligned}
$$

and

$$
\begin{aligned}
x_{j} \theta_{x_{i}}\left(\theta_{x_{j}}+\beta_{j}\right) u(t) & =x_{j} \partial_{t_{j}}\left\{t_{j} \theta_{x_{i}} u(t)\right\}+x_{j} \frac{\lambda_{0} t_{j}}{1-\sum_{1 \leq s \leq n} t_{s}} \theta_{x_{i}} u(t) \\
& =x_{j} \partial_{t_{j}}\left\{t_{j} \theta_{x_{i}} u(t)\right\}+x_{j} \frac{\lambda_{0} t_{j}}{1-\sum_{1 \leq s \leq n} t_{s}} \frac{\alpha t_{i} x_{i}}{1-\sum_{1 \leq s \leq n} x_{s} t_{s}} u(t),
\end{aligned}
$$

which lead to

$$
\begin{aligned}
& {\left[x_{i} \theta_{x_{j}}\left(\theta_{x_{i}}+\beta_{i}\right)-x_{j} \theta_{x_{i}}\left(\theta_{x_{j}}+\beta_{j}\right)\right] u(t)} \\
& \quad=x_{i} \partial_{t_{i}}\left\{t_{i} \theta_{x_{j}} u(t)\right\}-x_{j} \partial_{t_{j}}\left\{t_{j} \theta_{x_{i}} u(t)\right\}, \quad 1 \leq i \neq j \leq n,
\end{aligned}
$$

and thus

$$
\left[x_{i} \theta_{x_{j}}\left(\theta_{x_{i}}+\beta_{i}\right)-x_{j} \theta_{x_{i}}\left(\theta_{x_{j}}+\beta_{j}\right)\right] \int_{C} u(t) d t=0, \quad 1 \leq i \neq j \leq n,
$$

for a cycle $C$. This completes the proof.

Remark 5.6. If $\beta_{1}, \ldots, \beta_{n}, \gamma-\beta_{1}-\cdots-\beta_{n} \notin \mathbb{Z}$, then we have

$$
\begin{aligned}
& \int_{\operatorname{reg}\left(t_{1}>0, \ldots, t_{n}>0,1>t_{1}+\cdots+t_{n}\right)} \\
& \quad \times \prod_{s=1}^{n} t_{s}^{\beta_{s}-1}\left(1-\sum_{s=1}^{n} t_{s}\right)^{\gamma-1-\sum_{1 \leq s \leq n} \beta_{s}}\left(1-\sum_{s=1}^{n} t_{s} x_{s}\right)^{-\alpha} d t_{1} \cdots d t_{n} \\
& =\frac{\Gamma\left(\beta_{1}\right) \cdots \Gamma\left(\beta_{n}\right) \Gamma\left(\gamma-\beta_{1}-\cdots-\beta_{n}\right)}{\Gamma(\gamma)} \times F_{D}\left(\alpha, \beta_{1}, \ldots, \beta_{n}, \gamma ; x_{1}, \ldots, x_{n}\right),
\end{aligned}
$$

where the argument of each factor of the integrand is fixed to be zero when $x_{1}, \ldots, x_{n}$ are small positive numbers. This formula is given in Lauricella $[\mathbf{L}]$.

\section{Generalized hypergeometric equation ${ }_{n+1} E_{n}$}

\subsection{Integrals of difference products}

Let $x$ be a point of $\mathbb{C} \backslash\{0,1\}$, and let $u(t)=u(x ; t)$ be a multivalued function

$$
u(t)=\prod_{i=1}^{n} t_{i}^{\lambda_{i}} \prod_{i=1}^{n+1}\left(t_{i-1}-t_{i}\right)^{\lambda_{i-1, i}} \quad \text { with } t_{0}=1, t_{n+1}=x,
$$


on

$$
T_{x}=\mathbb{C}^{n} \backslash \bigcup_{i=1}^{n}\left\{t_{i}=0\right\} \cup \bigcup_{i=1}^{n+1}\left\{t_{i-1}-t_{i}=0\right\},
$$

where

$$
\lambda_{i}=\alpha_{i+1}-\beta_{i}, \quad \lambda_{i-1, i}=\beta_{i}-\alpha_{i}-1, \quad \text { and } \quad \beta_{n+1}=1 .
$$

Let $\mathcal{L}_{x}^{\vee}$ be the locally constant sheaf (local system) determined by $u(t)$.

Then we obtain Theorem 6.1.

THEOREM 6.1. We have

$$
\left[\theta_{x} \prod_{s=1}^{n}\left(\theta_{x}+\beta_{s}-1\right)-x \prod_{s=1}^{n+1}\left(\theta_{x}+\alpha_{s}\right)\right] \int_{C} u(t) d t=0
$$

for any cycle $C \in H_{n}\left(T_{x}, \mathcal{L}_{x}^{\vee}\right)$.

Proof. Direct calculation shows that

$$
\begin{aligned}
\theta_{x} u(t) & =\frac{-\lambda_{n, n+1} x}{t_{n}-x} u(t), \\
\theta_{t_{j}} u(t) & =t_{j}\left(\frac{\lambda_{j}}{t_{j}}+\frac{\lambda_{j, j+1}}{t_{j}-t_{j+1}}-\frac{\lambda_{j-1, j}}{t_{j-1}-t_{j}}\right) u(t) \\
& =\left(\lambda_{j}+\frac{\lambda_{j, j+1} t_{j}}{t_{j}-t_{j+1}}-\frac{\lambda_{j-1, j} t_{j}}{t_{j-1}-t_{j}}\right) u(t), \quad j=1, \ldots, n .
\end{aligned}
$$

Since $-x=\left(t_{n}-x\right)-t_{n},(6.2)$ can be written as

$$
\theta_{x} u(t)=\left(\lambda_{n, n+1}+\frac{-\lambda_{n, n+1} t_{n}}{t_{n}-x}\right) u(t) .
$$

Since $t_{j}=\left(t_{j}-t_{j+1}\right)+t_{j+1}$ or $-t_{j}=\left(-t_{j}+t_{j-1}\right)-t_{j-1}$, (6.3) can be written as

$$
\theta_{t_{j}} u(t)=\left(\lambda_{j}+\lambda_{j, j+1}+\frac{\lambda_{j, j+1} t_{j+1}}{t_{j}-t_{j+1}}-\frac{\lambda_{j-1, j} t_{j}}{t_{j-1}-t_{j}}\right) u(t), \quad j=1, \ldots, n,
$$

or

$$
\theta_{t_{j}} u(t)=\left(\lambda_{j}+\lambda_{j-1, j}+\frac{\lambda_{j, j+1} t_{j}}{t_{j}-t_{j+1}}-\frac{\lambda_{j-1, j} t_{j-1}}{t_{j-1}-t_{j}}\right) u(t), \quad j=1, \ldots, n .
$$

Summing both sides of (6.2) and (6.5) for $s \leq j \leq n$, we get

$$
\left(\theta_{x}+\sum_{j=s}^{n} \theta_{t_{j}}\right) u(t)=\left(\sum_{j=s}^{n}\left(\lambda_{j}+\lambda_{j, j+1}\right)-\frac{\lambda_{s-1, s} t_{s}}{t_{s-1}-t_{s}}\right) u(t), \quad s=1, \ldots, n .
$$

Summing both sides of (6.4) and (6.6) for $s \leq j \leq n$, we get

$$
\left(\theta_{x}+\sum_{j=s}^{n} \theta_{t_{j}}\right) u(t)=\left(\lambda_{n, n+1}+\sum_{j=s}^{n}\left(\lambda_{j}+\lambda_{j-1, j}\right)-\frac{\lambda_{s-1, s} t_{s-1}}{t_{s-1}-t_{s}}\right) u(t), \quad s=1, \ldots, n .
$$


Since $\theta_{t_{j}}=\partial_{t_{j}} t_{j}-1$ as an operator, equalities (6.7) and (6.8) can be written as

$$
\begin{gathered}
\left(\theta_{x}+\sum_{j=s}^{n} \partial_{t_{j}} t_{j}-(n-s+1)-\sum_{j=s}^{n}\left(\lambda_{j}+\lambda_{j, j+1}\right)\right) u(t) \\
=-\frac{\lambda_{s-1, s} t_{s}}{t_{s-1}-t_{s}} u(t), \quad s=1, \ldots, n,
\end{gathered}
$$

and

$$
\begin{gathered}
\left(\theta_{x}+\sum_{j=s}^{n} \partial_{t_{j}} t_{j}-(n-s+1)-\lambda_{n, n+1}-\sum_{j=s}^{n}\left(\lambda_{j}+\lambda_{j-1, j}\right)\right) u(t) \\
=-\frac{\lambda_{s-1, s} t_{s-1}}{t_{s-1}-t_{s}} u(t), \quad s=1,2, \ldots, n .
\end{gathered}
$$

Considering the equality (6.9) multiplied by $\prod_{j=1}^{s-1}\left(t_{j} /\left(t_{j-1}-t_{j}\right)\right)$ and the equality (6.10) multiplied by $\prod_{j=1}^{s-1}\left(t_{j-1} /\left(t_{j-1}-t_{j}\right)\right)$, we have

$$
\begin{aligned}
& \left(\theta_{x}-(n-s+1)-\sum_{j=s}^{n}\left(\lambda_{j}+\lambda_{j, j+1}\right)\right) \int_{C} \prod_{j=1}^{s-1} \frac{t_{j}}{t_{j-1}-t_{j}} u(t) d t \\
& =-\lambda_{s-1, s} \int_{C} \prod_{j=1}^{s} \frac{t_{j}}{t_{j-1}-t_{j}} u(t) d t, \quad s=1, \ldots, n,
\end{aligned}
$$

and

$$
\begin{aligned}
& \left(\theta_{x}-(n-s+1)-\lambda_{n, n+1}-\sum_{j=s}^{n}\left(\lambda_{j}+\lambda_{j-1, j}\right)\right) \int_{C} \prod_{j=1}^{s-1} \frac{t_{j-1}}{t_{j-1}-t_{j}} u(t) d t \\
& =-\lambda_{s-1, s} \int_{C} \prod_{j=1}^{s} \frac{t_{j-1}}{t_{j-1}-t_{j}} u(t) d t, \quad s=1, \ldots, n,
\end{aligned}
$$

for a cycle $C$. Successive application of (6.11) and (6.12) implies

$$
\begin{aligned}
& \prod_{s=1}^{n}\left(\theta_{x}-(n-s+1)-\sum_{j=s}^{n}\left(\lambda_{j}+\lambda_{j, j+1}\right)\right) \int_{C} u(t) d t \\
& \quad=\prod_{s=2}^{n}\left(\theta_{x}-(n-s+1)-\sum_{j=s}^{n}\left(\lambda_{j}+\lambda_{j, j+1}\right)\right)\left(-\lambda_{0,1}\right) \int_{C} \frac{t_{1}}{t_{0}-t_{1}} u(t) d t \\
& \quad=\prod_{s=3}^{n}\left(\theta_{x}-(n-s+1)-\sum_{j=s}^{n}\left(\lambda_{j}+\lambda_{j, j+1}\right)\right)\left(-\lambda_{0,1}\right)\left(-\lambda_{1,2}\right) \int_{C} \prod_{j=1}^{2} \frac{t_{j}}{t_{j-1}-t_{j}} u(t) d t \\
& =\ldots \\
& \quad=\prod_{s=1}^{n}\left(-\lambda_{s-1, s}\right) \int_{C} \prod_{j=1}^{n} \frac{t_{j}}{t_{j-1}-t_{j}} u(t) d t,
\end{aligned}
$$


and

$$
\begin{aligned}
\prod_{s=1}^{n} & \left(\theta_{x}-(n-s+1)-\lambda_{n, n+1}-\sum_{j=s}^{n}\left(\lambda_{j}+\lambda_{j-1, j}\right)\right) \int_{C} u(t) d t \\
= & \prod_{s=2}^{n}\left(\theta_{x}-(n-s+1)-\lambda_{n, n+1}-\sum_{j=s}^{n}\left(\lambda_{j}+\lambda_{j-1, j}\right)\right)\left(-\lambda_{0,1}\right) \int_{C} \frac{t_{0}}{t_{0}-t_{1}} u(t) d t \\
= & \prod_{s=3}^{n}\left(\theta_{x}-(n-s+1)-\lambda_{n, n+1}-\sum_{j=s}^{n}\left(\lambda_{j}+\lambda_{j-1, j}\right)\right)\left(-\lambda_{0,1}\right)\left(-\lambda_{1,2}\right) \\
& \times \int_{C} \prod_{j=1}^{2} \frac{t_{j-1}}{t_{j-1}-t_{j}} u(t) d t \\
= & \ldots \\
= & \prod_{s=1}^{n}\left(-\lambda_{s-1, s}\right) \int_{C} \prod_{j=1}^{n} \frac{t_{j-1}}{t_{j-1}-t_{j}} u(t) d t .
\end{aligned}
$$

On the other hand, equality (6.2) multiplied by $\prod_{j=1}^{n}\left(t_{j} /\left(t_{j-1}-t_{j}\right)\right)$ leads to

$$
\theta_{x} \int_{C} \prod_{j=1}^{n} \frac{t_{j}}{t_{j-1}-t_{j}} u(t) d t=\left(-\lambda_{n, n+1}\right) \int_{C} \prod_{j=1}^{n+1} \frac{t_{j}}{t_{j-1}-t_{j}} u(t) d t
$$

and equality (6.4) multiplied by $\prod_{j=1}^{n}\left(t_{j-1} /\left(t_{j-1}-t_{j}\right)\right)$ leads to

$$
\left(\theta_{x}-\lambda_{n, n+1}\right) \int_{C} \prod_{j=1}^{n} \frac{t_{j-1}}{t_{j-1}-t_{j}} u(t) d t=\left(-\lambda_{n, n+1}\right) \int_{C} \prod_{j=1}^{n+1} \frac{t_{j-1}}{t_{j-1}-t_{j}} u(t) d t
$$

Therefore, combination of (6.13) with (6.15) and combination of (6.14) with (6.16) imply

$$
\begin{gathered}
\theta_{x} \prod_{s=1}^{n}\left(\theta_{x}-(n-s+1)-\sum_{j=s}^{n}\left(\lambda_{j}+\lambda_{j, j+1}\right)\right) \int_{C} u(t) d t \\
=\prod_{s=1}^{n+1}\left(-\lambda_{s-1, s}\right) \int_{C} \prod_{j=1}^{n+1} \frac{t_{j}}{t_{j-1}-t_{j}} u(t) d t
\end{gathered}
$$

and

$$
\begin{gathered}
\left(\theta_{x}-\lambda_{n, n+1}\right) \prod_{s=1}^{n}\left(\theta_{x}-(n-s+1)-\lambda_{n, n+1}-\sum_{j=s}^{n}\left(\lambda_{j}+\lambda_{j-1, j}\right)\right) \int_{C} u(t) d t \\
=\prod_{s=1}^{n+1}\left(-\lambda_{s-1, s}\right) \int_{C} \prod_{j=1}^{n+1} \frac{t_{j-1}}{t_{j-1}-t_{j}} u(t) d t,
\end{gathered}
$$


which lead to

$$
\begin{aligned}
& {\left[\theta_{x} \prod_{s=1}^{n}\left(\theta_{x}-(n-s+1)-\sum_{j=s}^{n}\left(\lambda_{j}+\lambda_{j, j+1}\right)\right)\right.} \\
& \left.-x\left(\theta_{x}-\lambda_{n, n+1}\right) \prod_{s=1}^{n}\left(\theta_{x}-(n-s+1)-\lambda_{n, n+1}-\sum_{j=s}^{n}\left(\lambda_{j}+\lambda_{j-1, j}\right)\right)\right] \int_{C} u(t) d t .
\end{aligned}
$$

Since

$$
\begin{aligned}
\sum_{j=s}^{n}\left(\lambda_{j}+\lambda_{j, j+1}\right) & =\beta_{n+1}-\beta_{s}-(n-s+1), \\
\lambda_{n, n+1}+\sum_{j=s}^{n}\left(\lambda_{j}+\lambda_{j-1, j}\right) & =-\alpha_{s}-(n-s+1),
\end{aligned}
$$

which follow from (6.1), we reach the result.

Remark 6.2. If we assume the condition for the exponents

$$
\alpha_{i}-\beta_{j} \notin \mathbb{Z}, 1 \leq i, j \leq n+1, \quad \text { with } \beta_{n+1}=1,
$$

then the rank of $H_{n}\left(T_{x}, \mathcal{L}_{x}^{\vee}\right)$ is $n+1$, and a set of $n+1$ cycles gives rise to a fundamental set of solutions of ${ }_{n+1} E_{n}$. Indeed, such a set of cycles is given in [M1] and [M3].

Remark 6.3. It is known that ${ }_{n+1} E_{n}$ is irreducible if and only if (6.17) holds. See [BH] and [M3].

Remark 6.4. If $\alpha_{j}, \beta_{j}-\alpha_{j} \notin \mathbb{Z}, j=1, \ldots, n$, then we have

$$
\begin{gathered}
\int_{\operatorname{reg}\left(1<t_{1}<\cdots<t_{n}<\infty\right)} \prod_{s=1}^{n}\left\{t_{s}^{\alpha_{s+1}-\beta_{s}}\left(t_{s}-t_{s-1}\right)^{\beta_{s}-\alpha_{s}-1}\right\}\left(t_{n}-x\right)^{-\alpha_{n+1}} d t_{1} \cdots d t_{n} \\
\quad=\prod_{j=1}^{n} \frac{\Gamma\left(\alpha_{j}\right) \Gamma\left(\beta_{j}-\alpha_{j}\right)}{\Gamma\left(\beta_{j}\right)} \times{ }_{n+1} F_{n}\left(\begin{array}{c}
\alpha_{1}, \alpha_{2}, \ldots, \alpha_{n+1} \\
\beta_{1}, \ldots \ldots, \beta_{n}
\end{array} ; x\right),
\end{gathered}
$$

where $t_{0}=1$ and the argument of each factor of the integrand is fixed to be zero when $0<x<1$. This expression is given in (109) of [Po]. See also [A] and [M2].

\subsection{Integrals including a polynomial of degree $n$}

Let $x$ be a point of $\mathbb{C} \backslash\{0,1\}$, and let $u(t)=u(x ; t)$ be a multivalued function

$$
u(t)=\left(1-t_{1} t_{2} \cdots t_{n} x\right)^{-\alpha_{n+1}} \prod_{i=1}^{n} t_{i}^{\alpha_{i}-1}\left(1-t_{i}\right)^{\beta_{i}-\alpha_{i}-1}
$$

on

$$
T_{x}=\mathbb{C}^{n} \backslash \bigcup_{i=1}^{n}\left\{t_{i}=0\right\} \cup \bigcup_{i=1}^{n}\left\{t_{i}=1\right\} \cup\left\{t_{1} t_{2} \cdots t_{n} x=1\right\} .
$$

Let $\mathcal{L}_{x}^{\vee}$ be the locally constant sheaf (local system) determined by $u(t)$.

Then we obtain Theorem 6.5. 
THEOREM 6.5. We have

$$
\left[\theta_{x} \prod_{s=1}^{n}\left(\theta_{x}+\beta_{s}-1\right)-x \prod_{s=1}^{n+1}\left(\theta_{x}+\alpha_{s}\right)\right] \int_{C} u(t) d t=0
$$

for any cycle $C \in H_{n}\left(T_{x}, \mathcal{L}_{x}^{\vee}\right)$.

Proof. Direct calculation shows that

$$
\begin{aligned}
\theta_{x} u(t) & =\frac{\alpha_{n+1} t_{1} t_{2} \cdots t_{n} x}{1-t_{1} t_{2} \cdots t_{n} x} u(t), \\
\theta_{t_{i}} u(t) & =\left[\frac{\alpha_{n+1} t_{1} t_{2} \cdots t_{n} x}{1-t_{1} t_{2} \cdots t_{n} x}+\left(\alpha_{i}-1\right)+\frac{\left(\beta_{i}-\alpha_{i}-1\right) t_{i}}{1-t_{i}}\right] u(t), \quad i=1, \ldots, n .
\end{aligned}
$$

Since $t_{1} \cdots t_{n} x=1-\left(1-t_{1} \cdots t_{n} x\right)$, (6.19) can be written as

$$
\left(\theta_{x}+\alpha_{n+1}\right) u(t)=\frac{\alpha_{n+1}}{1-t_{1} t_{2} \cdots t_{n} x} u(t) .
$$

From (6.19) and (6.20), it follows that

$$
\left(\theta_{x}-\theta_{t_{i}}\right) u(t)=\left[1-\alpha_{i}+\frac{\left(\beta_{i}-\alpha_{i}-1\right) t_{i}}{1-t_{i}}\right] u(t),
$$

which is equivalent to

$$
\left(\theta_{x}+\alpha_{i}\right) u(t)=\partial_{t_{i}}\left\{t_{i} u(t)\right\}+\frac{\left(\beta_{i}-\alpha_{i}-1\right) t_{i}}{1-t_{i}} u(t),
$$

since $\theta_{t_{i}}=-1+\partial_{t_{i}} t_{i}$, as an operator. Moreover, (6.22) can be written as

$$
\left(\theta_{x}+\beta_{i}-1\right) u(t)=\partial_{t_{i}}\left\{t_{i} u(t)\right\}+\frac{\left(\beta_{i}-\alpha_{i}-1\right)}{1-t_{i}} u(t),
$$

since $t_{i}=1-\left(1-t_{i}\right)$. Successive application of (6.22) implies

$$
\prod_{i=1}^{n}\left(\theta_{x}+\alpha_{i}\right) \int_{C} u(t) d t=\prod_{i=1}^{n}\left(\beta_{i}-\alpha_{i}-1\right) \int_{C} \prod_{i=1}^{n} \frac{t_{i}}{1-t_{i}} u(t) d t,
$$

and application of $\left(\theta_{x}-\alpha_{n+1}\right)$ by (6.21) induces

$$
\prod_{i=1}^{n+1}\left(\theta_{x}+\alpha_{i}\right) \int_{C} u(t) d t=\prod_{i=1}^{n}\left(\beta_{i}-\alpha_{i}-1\right) \int_{C} \frac{\alpha_{n+1}}{1-t_{1} t_{2} \cdots t_{n} x} \prod_{i=1}^{n} \frac{t_{i}}{1-t_{i}} u(t) d t
$$

for any cycle $C \in H_{n}\left(T_{x}, \mathcal{L}_{x}^{\vee}\right)$. Similarly, successive application of (6.23) and application of $\theta_{x}$ by (6.19) imply

$$
\theta_{x} \prod_{i=1}^{n}\left(\theta_{x}-\beta_{i}-1\right) \int_{C} u(t) d t=\prod_{i=1}^{n}\left(\beta_{i}-\alpha_{i}-1\right) \int_{C} \frac{\alpha_{n+1} t_{1} \cdots t_{n} x}{1-t_{1} t_{2} \cdots t_{n} x} \prod_{i=1}^{n} \frac{1}{1-t_{i}} u(t) d t .
$$

Combining (6.24) and (6.25), we get

$$
\left[x \prod_{i=1}^{n+1}\left(\theta_{x}+\alpha_{i}\right)-\theta_{x} \prod_{i=1}^{n}\left(\theta_{x}-\beta_{i}-1\right)\right] \int_{C} u(t) d t=0 .
$$

This completes the proof. 
Remark 6.6. If $\alpha_{j}, \beta_{j}-\alpha_{j} \notin \mathbb{Z}, j=1, \ldots, n$, then we have

$$
\begin{gathered}
\int_{\operatorname{reg}(0,1)^{n}}\left(1-t_{1} t_{2} \cdots t_{n} x\right)^{-\alpha_{n+1}} \prod_{i=1}^{n} t_{i}^{\alpha_{i}-1}\left(1-t_{i}\right)^{\beta_{i}-\alpha_{i}-1} d t_{1} \cdots d t_{n} \\
=\prod_{j=1}^{n} \frac{\Gamma\left(\alpha_{j}\right) \Gamma\left(\beta_{j}-\alpha_{j}\right)}{\Gamma\left(\beta_{j}\right)} \times{ }_{n+1} F_{n}\left(\begin{array}{c}
\alpha_{1}, \alpha_{2}, \ldots, \alpha_{n+1} \\
\beta_{1}, \ldots \ldots, \beta_{n}
\end{array} ; x\right),
\end{gathered}
$$

where the argument of each factor of the integrand is fixed to be zero when $0<x<1$. This expression is given on [Go, p. 281], on [Po, p. 78], and is derived from [W, equations (38) and (38a)]. See also [A].

Remark 6.7. By the change of integration variables such that $t_{1} \cdots t_{j}=1 / u_{j}, j=1, \ldots, n$, we obtain the left-hand side of (6.18) from the left-hand side of (6.26).

\section{Hypergeometric system $R E(k, n)$ on the configuration space}

Let $u(t)=u(x ; t)$ be a multivalued function

$$
u(x ; t)=\left\{\prod_{s=2}^{k} t_{s}^{\lambda_{s}}\right\}\left(1-\sum_{s=2}^{k} t_{s}\right)^{\lambda_{k+1}}\left\{\prod_{p=k+2}^{n}\left(1-\sum_{s=2}^{k} t_{s} x_{s p}\right)^{\lambda_{p}}\right\}
$$

on

$$
T_{x}=\mathbb{C}^{k-1} \backslash \bigcup_{s=2}^{k}\left\{t_{s}=0\right\} \cup\left\{\sum_{s=2}^{k} t_{s}=1\right\} \cup \bigcup_{p=k+2}^{n}\left\{\sum_{s=2}^{k} t_{s} x_{s p}=1\right\} \subset\left(\mathbb{P}^{1}\right)^{k-1}
$$

Let $\mathcal{L}_{x}^{\vee}$ be the locally constant sheaf (local system) determined by $u(t)$.

Then we get Theorem 7.1.

THEOREM 7.1. We have

$$
\begin{aligned}
& {\left[x_{i p}\left(\theta_{i}+\lambda_{i}+1\right)\left(\theta^{p}-\lambda_{p}\right)-\theta_{i p}\left(\theta+\lambda_{0}-1\right)\right] \int_{C} u(t) d t=0,} \\
& 2 \leq i \leq k \text { and } k+2 \leq p \leq n, \\
& {\left[x_{i q} \theta_{i p}\left(\theta^{q}-\lambda_{q}\right)-x_{i p} \theta_{i q}\left(\theta^{p}-\lambda_{p}\right)\right] \int_{C} u(t) d t=0, \quad 2 \leq i \leq k \text { and } k+2 \leq p, q \leq n,} \\
& {\left[x_{i p} \theta_{j p}\left(\theta_{i}+\lambda_{i}+1\right)-x_{j p} \theta_{i p}\left(\theta_{j}+\lambda_{j}+1\right)\right] \int_{C} u(t) d t=0,} \\
& \quad 2 \leq i, j \leq k \text { and } k+2 \leq p \leq n, \\
& {\left[x_{i q} x_{j p} \theta_{i p} \theta_{j q}-x_{i p} x_{j q} \theta_{i q} \theta_{j p}\right] \int_{C} u(t) d t=0, \quad 2 \leq i, j \leq k \text { and } k+2 \leq p, q \leq n,}
\end{aligned}
$$


for any cycle $C \in H_{k-1}\left(T_{x}, \mathcal{L}_{x}^{\vee}\right)$, where

$$
\begin{aligned}
\lambda_{0} & =\lambda_{2}+\cdots+\lambda_{k+1}+k, \\
\theta_{j q} & =x_{j q} \frac{\partial}{\partial x_{j q}}, \\
\theta_{j} & =\theta_{j, k+2}+\theta_{j, k+3}+\cdots+\theta_{j, n}, \\
\theta^{q} & =\theta_{2, q}+\theta_{3, q}+\cdots+\theta_{k, q}, \\
\theta & =\theta^{k+2}+\cdots+\theta^{n}=\theta_{2}+\cdots+\theta_{k}=\sum_{\substack{2 \leq i \leq k \\
k+2 \leq r \leq n}} \theta_{i r} .
\end{aligned}
$$

Proof. Direct calculation shows that

$$
\begin{aligned}
\theta_{x_{i p}} u(t) & =\frac{-\lambda_{p} t_{i} x_{i p}}{1-\sum_{2 \leq s \leq k} t_{s} x_{s p}} u(t), \\
\theta_{t_{i}} u(t) & =\left[\lambda_{i}+\frac{-\lambda_{k+1} t_{i}}{1-\sum_{2 \leq s \leq k} t_{s}}+\sum_{p=k+2}^{n} \frac{-\lambda_{p} t_{i} x_{i p}}{1-\sum_{2 \leq s \leq k} t_{s} x_{s p}}\right] u(t)
\end{aligned}
$$

for $2 \leq i \leq k, k+2 \leq p \leq n$. Summing both sides of (7.2) over $i$, we have

$$
\left(\sum_{2 \leq i \leq k} \theta_{x_{i p}}-\lambda_{p}\right) u(t)=\frac{-\lambda_{p}}{1-\sum_{2 \leq s \leq k} t_{s} x_{s p}} u(t)
$$

since $\sum_{s} t_{s} x_{s p}=1-\left(1-\sum_{s} t_{s} x_{s p}\right)$.

It follows from (7.2) and (7.3) that

$$
\left(\sum_{k+2 \leq p \leq n} \theta_{x_{i p}}-\theta_{t_{i}}\right) u(t)=\left[-\lambda_{i}+\frac{\lambda_{k+1} t_{i}}{1-\sum_{2 \leq s \leq k} t_{s}}\right] u(t),
$$

which is equivalent to

$$
\left(\sum_{k+2 \leq p \leq n} \theta_{x_{i p}}+\lambda_{i}+1\right) u(t)=\partial_{t_{i}}\left\{t_{i} u(t)\right\}+\frac{\lambda_{k+1} t_{i}}{1-\sum_{2 \leq s \leq k} t_{s}} u(t),
$$

since $\theta_{t_{i}}=-1+\partial_{t_{i}} t_{i}$, as an operator. Moreover, summing both sides of (7.5) over $i$, we have

$$
\left(\sum_{2 \leq i \leq k} \sum_{k+2 \leq p \leq n} \theta_{x_{i p}}+\sum_{2 \leq i \leq k} \lambda_{i}+(k-1)\right) u(t)=\sum_{2 \leq i \leq k} \partial_{t_{i}}\left\{t_{i} u(t)\right\}+\frac{\lambda_{k+1} \sum_{2 \leq i \leq k} t_{i}}{1-\sum_{2 \leq s \leq k} t_{s}} u(t),
$$

which is equivalent to

$$
\left(\sum_{2 \leq i \leq k} \sum_{k+2 \leq p \leq n} \theta_{x_{i p}}+\sum_{2 \leq i \leq k+1} \lambda_{i}+(k-1)\right) u(t)=\sum_{2 \leq i \leq k} \partial_{t_{i}}\left\{t_{i} u(t)\right\}+\frac{\lambda_{k+1}}{1-\sum_{2 \leq s \leq k} t_{s}} u(t),
$$

since $\sum_{2 \leq i \leq k} t_{i}=1-\left(1-\sum_{2 \leq i \leq k} t_{i}\right)$. 
By (7.4), the action of $\left(\sum_{2 \leq i \leq k} \theta_{x_{i p}}-\lambda_{p}\right)$ on (7.5) implies

$$
\begin{aligned}
& \left(\sum_{2 \leq t \leq k} \theta_{x_{t p}}-\lambda_{p}\right)\left(\sum_{k+2 \leq p^{\prime} \leq n} \theta_{x_{i p^{\prime}}}+\lambda_{i}+1\right) u(t) \\
& =\partial_{t_{i}}\left\{t_{i}\left(\sum_{2 \leq t \leq k} \theta_{x_{t p}}-\lambda_{p}\right) u(t)\right\}+\frac{\lambda_{k+1} t_{i}}{1-\sum_{2 \leq s \leq k} t_{s}}\left(\sum_{2 \leq t \leq k} \theta_{x_{t p}}-\lambda_{p}\right) u(t) \\
& =\partial_{t_{i}}\left\{t_{i} \frac{-\lambda_{p}}{1-\sum_{2 \leq s \leq k} t_{s} x_{s p}} u(t)\right\}+\frac{\lambda_{k+1} t_{i}}{1-\sum_{2 \leq s \leq k} t_{s}} \frac{-\lambda_{p}}{1-\sum_{2 \leq s \leq k} t_{s} x_{s p}} u(t) .
\end{aligned}
$$

By (7.2), the action of $\theta_{x_{i p}}$ on (7.6) implies

$$
\begin{aligned}
& \theta_{x_{i p}}\left(\sum_{2 \leq i^{\prime} \leq k} \sum_{k+2 \leq p^{\prime} \leq n} \theta_{x_{i^{\prime} p^{\prime}}}+\sum_{2 \leq i^{\prime} \leq k+1} \lambda_{i^{\prime}}+(k-1)\right) u(t) \\
& =\sum_{2 \leq i^{\prime} \leq k} \partial_{t_{i^{\prime}}}\left\{t_{i^{\prime}} \theta_{x_{i p}} u(t)\right\}+\frac{\lambda_{k+1}}{1-\sum_{2 \leq s \leq k} t_{s}} \theta_{x_{i p}} u(t) \\
& =\sum_{2 \leq i^{\prime} \leq k} \partial_{t_{i^{\prime}}}\left\{t_{i^{\prime}} \frac{-\lambda_{p} t_{i} x_{i p}}{1-\sum_{2 \leq s \leq k} t_{s} x_{s p}} u(t)\right\}+\frac{\lambda_{k+1}}{1-\sum_{2 \leq s \leq k} t_{s}} \frac{-\lambda_{p} t_{i} x_{i p}}{1-\sum_{2 \leq s \leq k} t_{s} x_{s p}} u(t) .
\end{aligned}
$$

Combining (7.7) and (7.8), we obtain

$$
\begin{aligned}
& {\left[x_{i p}\left(\sum_{2 \leq t \leq k} \theta_{x_{t p}}-\lambda_{p}\right)\left(\sum_{k+2 \leq p^{\prime} \leq n} \theta_{x_{i p^{\prime}}}+\lambda_{i}+1\right)\right.} \\
& \left.\quad-\theta_{x_{i p}}\left(\sum_{2 \leq i^{\prime} \leq k} \sum_{k+2 \leq p^{\prime} \leq n} \theta_{x_{i^{\prime} p^{\prime}}}+\sum_{2 \leq i^{\prime} \leq k+1} \lambda_{i^{\prime}}+(k-1)\right)\right] u(t) \\
& \quad=x_{i p} \partial_{t_{i}}\left\{t_{i} \frac{-\lambda_{p}}{1-\sum_{2 \leq s \leq k} t_{s} x_{s p}} u(t)\right\}-\sum_{2 \leq i^{\prime} \leq k} \partial_{t_{i^{\prime}}}\left\{t_{i^{\prime}} \frac{-\lambda_{p} t_{i} x_{i p}}{1-\sum_{2 \leq s \leq k} t_{s} x_{s p}} u(t)\right\},
\end{aligned}
$$

which implies

$$
\begin{aligned}
& {\left[x_{i p}\left(\sum_{2 \leq t \leq k} \theta_{x_{t p}}-\lambda_{p}\right)\left(\sum_{k+2 \leq p^{\prime} \leq n} \theta_{x_{i p^{\prime}}}+\lambda_{i}+1\right)\right.} \\
& \left.-\theta_{x_{i p}}\left(\sum_{2 \leq i^{\prime} \leq k} \sum_{k+2 \leq p^{\prime} \leq n} \theta_{x_{i^{\prime} p^{\prime}}}+\lambda_{0}-1\right)\right] \int_{C} u(t) d t=0 .
\end{aligned}
$$

Next, the action of $\theta_{x_{i p}}$ on (7.4) by (7.2) implies

$$
\theta_{x_{i p}}\left(\sum_{2 \leq i^{\prime} \leq k} \theta_{x_{i^{\prime} q}}-\lambda_{q}\right) u(t)=\frac{-\lambda_{q}}{1-\sum_{2 \leq s \leq k} t_{s} x_{s q}} \frac{-\lambda_{p} t_{i} x_{i p}}{1-\sum_{2 \leq s \leq k} t_{s} x_{s p}} u(t)
$$

for $k+2 \leq p \neq q \leq n$, which leads to

$$
\left[x_{i q} \theta_{x_{i p}}\left(\sum_{2 \leq i^{\prime} \leq k} \theta_{x_{i^{\prime} q}}-\lambda_{q}\right)-x_{i p} \theta_{x_{i q}}\left(\sum_{2 \leq i^{\prime} \leq k} \theta_{x_{i^{\prime} p}}-\lambda_{p}\right)\right] u(t)=0,
$$


and

$$
\left[x_{i q} \theta_{x_{i p}}\left(\sum_{2 \leq i^{\prime} \leq k} \theta_{x_{i^{\prime} q}}-\lambda_{q}\right)-x_{i p} \theta_{x_{i q}}\left(\sum_{2 \leq i^{\prime} \leq k} \theta_{x_{i^{\prime} p}}-\lambda_{p}\right)\right] \int_{C} u(t) d t=0 .
$$

On the other hand, the action of $\theta_{x_{j p}}$ on (7.5) by (7.2) implies

$$
\begin{aligned}
\theta_{x_{j p}}\left(\sum_{k+2 \leq p^{\prime} \leq n} \theta_{x_{i p^{\prime}}}+\lambda_{i}+1\right) u(t)= & \partial_{t_{i}}\left\{t_{i} \theta_{x_{j p}} u(t)\right\}+\frac{\lambda_{k+1} t_{i}}{1-\sum_{2 \leq s \leq k} t_{s}} \theta_{x_{j p}} u(t) \\
= & \partial_{t_{i}}\left\{t_{i} \frac{-\lambda_{p} t_{j} x_{j p}}{1-\sum_{2 \leq s \leq k} t_{s} x_{s p}} u(t)\right\} \\
& +\frac{\lambda_{k+1} t_{i}}{1-\sum_{2 \leq s \leq k} t_{s}} \frac{-\lambda_{p} t_{i} x_{j p}}{1-\sum_{2 \leq s \leq k} t_{s} x_{s p}} u(t),
\end{aligned}
$$

which leads to

$$
\begin{gathered}
{\left[x_{i p} \theta_{x_{j p}}\left(\sum_{k+2 \leq p^{\prime} \leq n} \theta_{x_{i p^{\prime}}}+\lambda_{i}+1\right)-x_{j p} \theta_{x_{i p}}\left(\sum_{k+2 \leq p^{\prime} \leq n} \theta_{x_{j p^{\prime}}}+\lambda_{j}+1\right)\right] u(t)} \\
=x_{i p} \partial_{t_{i}}\left\{t_{i} \frac{-\lambda_{p} t_{j} x_{j p}}{1-\sum_{2 \leq s \leq k} t_{s} x_{s p}} u(t)\right\}-x_{j p} \partial_{t_{j}}\left\{t_{j} \frac{-\lambda_{p} t_{i} x_{i p}}{1-\sum_{2 \leq s \leq k} t_{s} x_{s p}} u(t)\right\},
\end{gathered}
$$

and

$$
\left[x_{i p} \theta_{x_{j p}}\left(\sum_{k+2 \leq p^{\prime} \leq n} \theta_{x_{i p^{\prime}}}+\lambda_{i}+1\right)-x_{j p} \theta_{x_{i p}}\left(\sum_{k+2 \leq p^{\prime} \leq n} \theta_{x_{j p^{\prime}}}+\lambda_{j}+1\right)\right] \int_{C} u(t) d t=0 .
$$

Finally, using (7.2) twice, we have, for $p \neq q$,

$$
\begin{aligned}
\theta_{x_{i p}} \theta_{x_{j q}} u(t) & =\theta_{x_{i p}} \frac{-\lambda_{q} t_{j} x_{j q}}{1-\sum_{2 \leq s \leq k} t_{s} x_{s q}} u(t)=\frac{-\lambda_{q} t_{i} x_{j q}}{1-\sum_{2 \leq s \leq k} t_{s} x_{s q}} \theta_{x_{i p}} u(t) \\
& =\frac{-\lambda_{q} t_{j} x_{j q}}{1-\sum_{2 \leq s \leq k} t_{s} x_{s q}} \frac{-\lambda_{p} t_{i} x_{i p}}{1-\sum_{2 \leq s \leq k} t_{s} x_{s q}} u(t),
\end{aligned}
$$

which leads to

$$
\left[x_{j p} x_{i q} \theta_{x_{i p}} \theta_{x_{j q}}-x_{j q} x_{i p} \theta_{x_{i q}} \theta_{x_{j p}}\right] u(t)=0,
$$

and

$$
\left[x_{j p} x_{i q} \theta_{x_{i p}} \theta_{x_{j q}}-x_{j q} x_{i p} \theta_{x_{i q}} \theta_{x_{j p}}\right] \int_{C} u(t) d t=0 .
$$

This completes the proof.

Remark 7.2. If $\lambda_{1}, \ldots, \lambda_{k+1} \notin \mathbb{Z}$, then we have

$$
\begin{aligned}
& \int_{\operatorname{reg}\left(t_{2}>0, \ldots, t_{k}>0,1>t_{2}+\cdots+t_{k}\right)}\left\{\prod_{s=2}^{k} t_{s}^{\lambda_{s}}\right\}\left(1-\sum_{s=2}^{k} t_{s}\right)^{\lambda_{k+1}} \prod_{p=k+2}^{n}\left(1-\sum_{s=2}^{k} t_{s} x_{s p}\right)^{\lambda_{p}} d t_{2} \cdots d t_{k} \\
& =\frac{\Gamma\left(\lambda_{2}+1\right) \cdots \Gamma\left(\lambda_{k+1}+1\right)}{\Gamma\left(\lambda_{2}+\cdots+\lambda_{k+1}+k\right)} \times F(k, n ; \lambda ; x),
\end{aligned}
$$

where the argument of each factor of the integrand is fixed to be zero when all $x_{s p}(2 \leq s \leq$ $k$ and $k+2 \leq p \leq n)$ are positive and small enough. See also [Ki, equation (8.5)]. 
Acknowledgements. The authors are grateful to Professors Jyoichi Kaneko, Takeshi Sasaki and Masaaki Yoshida for valuable comments, and also to the referee for a careful reading of the manuscript.

This work was supported by JSPS Grant-in-Aid for Scientific Research (B) 23340002 and (B) $15 \mathrm{H} 03626$.

\section{REFERENCES}

[A] K. Aomoto. Configurations and invariant theory of Gauss-Manin systems. Adv. Stud. Pure Math. 4 (1984), 165-179.

[AK] K. Aomoto and M. Kita. Theory of hypergeometric functions (Springer Monographs in Mathematics). Springer, Berlin, 2011.

[AKdF] P. Appell and J. Kempé de Fériet. Fonctions hypergéométriques et hypersphériques; Polynomes d'Hermite. Gauthier-Villars, Paris, 1926.

[BH] F. Beukers and G. Heckman. Monodromy for the hypergeometric function ${ }_{n} F_{n-1}$. Invent. Math. 95 (1989), 325-354.

[B] E. Bod. Algebraicity of the Appell-Lauricella and Horn hypergeometric functions. J. Differ. Equations 252 (2012), 541-566.

[C] K. Cho. A generalization of Kita and Noumi's vanishing theorems of cohomology groups of local system. Nagoya Math J. 147 (1997), 63-69.

[Ge] I. M. Gel'fand. General theory of hypergeometric functions. Soviet Math. Dokl. 33 (1986), 573-577.

[GKZ] I. M. Gel'fand, M. M. Kapranov and A. V. Zelevinskii. Generalized Euler integrals and A-hypergeometric functions. Adv. Math. 84 (1990), 255-271.

[Go] É. Goursat. Mémoire sur les fonctions hypergéométriques d'ordre supérieur. Ann. Sci. Éc. Norm. Supér. (2) 12 (1883), 261-286.

[Ho] E. Horikawa. Transformations and contiguity relations for Gelfand's hypergeometric functions. J. Math. Sci. Univ. Tokyo 1 (1994), 181-203.

[Ka] J. Kaneko. Monodromy group of Appell's system $\left(F_{4}\right)$. Tokyo J. Math. 4 (1981), 35-54.

[Ki] M. Kita. On hypergeometric functions in several variables. I. New integral representations of Euler type. Jpn. J. Math. 18 (1992), 25-74.

[KN] M. Kita and M. Noumi. On the structure of cohomology groups attached to the integral of certain many-valued analytic functions. Jpn. J. Math. 9 (1983), 113-157.

[L] G. Lauricella. Sulle Funzioni ipergeometriche a più variabili. Rend. Circ. Mat. Palermo 7 (1893), 111-158.

[MSY] K. Matsumoto, T. Sasaki and M. Yoshida. The monodromy of the period map of a 4-parameter family of K3 surfaces and the hypergeometric function of type (3, 6). Int. J. Math. 3 (1992), 1-164.

[M1] K. Mimachi. Intersection numbers for twisted cycles and the connection problem associated with the generalized hypergeometric function ${ }_{n+1} F_{n}$. Int. Math. Res. Not. IMRN 2011 (2011), 1757-1781.

[M2] K. Mimachi. Nakagiri's monodromy representations associated with Appell's hypergeometric functions $F_{2}$ and $F_{3}$. Funk. Ekvac. To appear.

[M3] K. Mimachi. Monodromy representations associated with the generalized hypergeometric function ${ }_{n+1} F_{n}$ and their reducibility. Kyushu J. Math. 70 (2016), 105-129.

[MS1] K. Mimachi and T. Sasaki. Irreducibility and reducibility of Lauricella's system of differential equations $E_{D}$ and the Jordan-Pochhammer differential equation $E_{J P}$. Kyushu J. Math. 66 (2012), 61-87.

[MS2] K. Mimachi and T. Sasaki. The hypergeometric systems and the contiguity operators on the Grassmannian manifold and on the associated configuration space. Int. J. Math. 27 (2016), 1650056.

[MY] K. Mimachi and M. Yoshida. Intersection numbers of twisted cycles and the correlation functions of the conformal field theory. Commun. Math. Phys. 234 (2003), 339-358.

[NN] M. Nakatani and M. Noumi. $q$-Hypergeometric systems arising from quantum Grassmannians. Funkt. Ekvac. 41 (1998), 363-381.

[Pa] P. I. Pastro. On the integral representation of $F_{4}$ of Appell and its Lauricella generalization. Bull. Sci. Math. 113 (1989), 119-124.

[Po] L. Pochhammer. Ueber die Differentialgleichung der allgemeineren hypergeometrischen Reihe mit zwei endlichen singulären Punkten. J. Reine Angew. Math. 102 (1888), 76-159.

[T] K. Takano. Integral representations of $F_{C}$. Private notes. 
[W] E. Winkler. Über die hypergeometrische differentialgleichung $n^{\text {ter }}$ ordnung mit zwei endlichen singulären punkten, Inaugural-Dissertation, Ludwig-Maximilians-Universität München, 1930.

[Y1] M. Yoshida. Euler integral transformations of hypergeometric functions of two variables. Hiroshima Math. J. 10 (1980), 329-335.

[Y2] M. Yoshida. Hypergeometric functions, my love: modular interpretations of configuration spaces. Vieweg, Wiesbaden, 1997.

\author{
Katsuhisa Mimachi \\ Department of Pure and Applied Mathematics \\ Osaka University \\ Suita, Osaka, 565-0871 \\ Japan \\ (E-mail:mimachi@math.sci.osaka-u.ac.jp) \\ Masatoshi Noumi \\ Department of Mathematics \\ Kobe University \\ Rokko, Kobe, 657-8501 \\ Japan \\ (E-mail:noumi@math.kobe-u.ac.jp)
}

